\title{
Energy Conservation, Singular Orbits, and FPGA Implementation of Two New Hamiltonian Chaotic Systems
}

\author{
Enzeng Dong $\mathbb{D}^{1},{ }^{1}$ Guanghan Liu $\mathbb{D},{ }^{1}$ Zenghui Wang $\mathbb{D}^{2},{ }^{2}$ and Zengqiang Chen $\mathbb{D}^{3}$ \\ ${ }^{1}$ Tianjin Key Laboratory For Control Theory \& Applications in Complicated Systems, Tianjin University of Technology, \\ Tianjin 300384, China \\ ${ }^{2}$ Department of Electrical and Mining Engineering, University of South Africa, Florida 1710, South Africa \\ ${ }^{3}$ Department of Automation, Nankai University, Tianjin 300071, China
}

Correspondence should be addressed to Enzeng Dong; dongenzeng@163.com and Guanghan Liu; 13821370371@163.com

Received 3 January 2020; Accepted 16 March 2020; Published 25 April 2020

Academic Editor: Sigurdur F. Hafstein

Copyright (c) 2020 Enzeng Dong et al. This is an open access article distributed under the Creative Commons Attribution License, which permits unrestricted use, distribution, and reproduction in any medium, provided the original work is properly cited.

\begin{abstract}
Since the conservative chaotic system (CCS) has no general attractors, conservative chaotic flows are more suitable for the chaosbased secure communication than the chaotic attractors. In this paper, two Hamiltonian conservative chaotic systems (HCCSs) are constructed based on the 4D Euler equations and a proposed construction method. These two new systems are investigated by equilibrium points, dynamical evolution map, Hamilton energy, and Casimir energy. They look similar, but it is found that one can be explained using Casimir power and another cannot be explained in terms of the mechanism of chaos. Furthermore, a pseudorandom signal generator is developed based on these proposed systems, which are tested based on NIST tests and implemented by using the field programmable gate array (FPGA) technique.
\end{abstract}

\section{Introduction}

Many dissipative chaotic systems have been widely studied in the past decades. Lorenz discovered the first chaos system named Lorenz system in 1963 [1]. In 1999, Chen and Ueta proposed a new chaotic attractor called Chen attractor, which is topologically more complex than Lorenz attractor [2]. Lü and Chen developed a new chaotic system named Lü system in 2002, which bridged the gap between the Lorenz system and the Chen system [3]. The complex dynamical behaviors of chaotic systems have been gradually excavated. Both conservative systems $[4,5]$ and dissipative systems [6-8] have some phenomena of transition from period to quasi-period and then into chaos. For the past few years, due to the potential applications of chaotic systems with complex dynamic behavior in speech signals and digital image/video encryption, analysis in this area has also become one of the main directions of chaos research $[9,10]$. Dong et al. rigorously verified the chaotic properties of a 3D autonomous four-wing chaotic attractor using topological horseshoe theory and numerical calculations $[11,12]$. As we know, the dissipative chaotic system (DCS) has strange attractors, which can be reconstructed by using the delay embedding method [13]. Compared with DCS, CCS has no general attractors, and the conservative chaotic flows have pseudorandomness and white-noise-like properties [14, 15]. Recently, there are some conservative chaotic systems that have been constructed and analyzed. Cang proposed several conservative chaotic systems with only linear and quadratic terms [16]. Soon afterwards, a new method for constructing high-dimensional conservative chaotic systems was proposed by Dong and Yuan, and a four-dimensional conservative chaotic system and a 5-dimensional conservative hyperchaotic system were specifically investigated [17].

Existing conservative systems can be divided into two categories: Hamiltonian conservative chaotic system (HCCS) and non-Hamiltonian CCS (non-HCCS). The HCCSs contain two subclasses: traditional HCCS [18] and generalized HCCS. The typical examples of traditional HCCSs are He'non-Heiles system (the maximum Lyapunov exponent $\mathrm{LE}_{1}=0.02$ ) [19] and quantum chaotic systems, and the classic generalized HCCSs are Cang-case A system 
$\left(\mathrm{LE}_{1}=1.2\right) \quad[20]$ and Qi system with large LEs $\left(\mathrm{LE}_{1}=78194.27\right)$ [21]. HCCS has zero-sum LEs and satisfies volume conservation $(\nabla \cdot f=0)$ and energy saving $(\dot{H}=0)$. However, non-HCCSs only meet the zero-sum LEs and do not need to satisfy the conditions of $\nabla \cdot f=0$ or $\dot{H}=0$. For example, Sprott A system $\left(\mathrm{LE}_{1}=0.014\right)$ has divergence $\nabla$. $f=x_{3}\left(x_{3} \in R\right)$ [22], and Vaidyanathan and Volos system $\left(\mathrm{LE}_{1}=0.0395\right)$ has $\nabla \cdot f=2 x_{3}[23]$ and the $6 \mathrm{D}$ conservative hyperchaotic system $\left(\mathrm{LE}_{1}=\mathrm{LE}_{2}=0.055\right)$ has $\nabla \cdot f=2 x_{5}$ [24]. As can be seen from the above classification and comparison, Hamiltonian conservative systems need to meet stricter condition than other conservative systems.

For the mechanism of chaos generation, Arnor'd first proposed a Kolmogorov system of using ordinary differential equations to describe a dissipative-forced dynamic system, and this system includes three field components: inertial, dissipative, and external components [25]. Afterwards, Qi transformed Qi four-wing chaotic system into a Kolmogorov-type system and then analyzed the cycling of energy among potential energy, kinetic energy, dissipation, and external energy [26]. Qi and Zhang and Wiggins [27, 28] found that the conservative part of the 3-dimensional Euler equations is symplectic structure in the generalized Hamiltonian system and the Kolmogorov system. Qi proposed six complete $4 \mathrm{D}$ Euler equations by extending a system from $3 \mathrm{D}$ to $4 \mathrm{D}[21]$.

However, how to construct a system conforming to the four-dimensional Euler equations has not been fully studied. Currently, some conservative chaotic systems are verified by analog circuits [29], while the digital circuit implementation of Hamiltonian conservative chaos is rarely reported. In this paper, two Hamiltonian conservative chaotic systems are constructed. We first select three linear functions and construct a four-dimensional system using the methods of Reference [17]. It accords with the conservative four-dimensional Euler equations of Hamilton and Casimir function, but the four-dimensional system does not produce chaos. Then, two linear terms are added based on the modified 4D Euler equations and a new constructive method. Choosing proper parameters and initial values, the constructed two systems show chaotic dynamics. For highdimensional systems, the equilibrium point analysis $[30,31]$ and bifurcation analysis [32] are necessary. In general, the noncanonical Hamiltonian systems have Casimir functions [33], but the structure matrix of the new two systems are different: one is noncanonical and another is canonical. Hence, we use two methods to analyze the mechanism of chaos generation. Pseudorandomness is confirmed following NIST statistic tests. In addition, the field programmable gate array technique is used to design pseudorandom number generators.

The rest of this article is organized as follows. In Section 2 , the construction of two new 4D HCCSs is given. In Section 3 , these two systems are analyzed using different theories. Section 4 contains the verification of strong pseudorandomness of these systems and a field programmable gate array-based digital pseudorandom number generator using the proposed HCCSs. Finally, the conclusions are drawn in Section 5.

\section{Modeling HCCS}

2.1. Preliminaries. Euler equation is the most important fundamental equation in the nonviscous fluid dynamics, and it is also very effective for describing rigid bodies with symmetric rotation [34].

$\Sigma_{24}$ are certain $4 \mathrm{D}$ Euler rigid body equations, which can be described as the form of a Hamiltonian vector field:

$$
\dot{x}=J_{24}(\mathbf{x}) \times \nabla H(\mathbf{x}),
$$

where

$$
J_{24}(\mathbf{x})=\left[\begin{array}{cccc}
0 & -x_{4} & 0 & x_{2} \\
x_{4} & 0 & -x_{4} & x_{3}-x_{1} \\
0 & x_{4} & 0 & -x_{2} \\
-x_{2} & x_{1}-x_{3} & x_{2} & 0
\end{array}\right],
$$

and $\nabla$ is the gradient operator. $H(\mathbf{x})$ is the Hamiltonian energy function.

$$
H(\mathbf{x})=\frac{1}{2}\left(\Pi_{1} x_{1}^{2}+\Pi_{2} x_{2}^{2}+\Pi_{3} x_{3}^{2}+\Pi_{4} x_{4}^{2}\right),
$$

where $\Pi_{i}=I_{i}^{-1}, I_{i}$ is the moment of inertia of the rigid body, and $x_{i}$ is the angular momentum. Then, system $\Sigma_{24}$ can be obtained as

$$
\left\{\begin{array}{l}
\dot{x}_{1}=\left(\Pi_{4}-\Pi_{2}\right) x_{2} x_{4} \\
\dot{x}_{2}=\left(\Pi_{1}-\Pi_{4}\right) x_{1} x_{4}+\left(\Pi_{4}-\Pi_{3}\right) x_{3} x_{4} \\
\dot{x}_{3}=\left(\Pi_{2}-\Pi_{4}\right) x_{2} x_{4} \\
\dot{x}_{4}=\left(\Pi_{2}-\Pi_{1}\right) x_{1} x_{2}+\left(\Pi_{3}-\Pi_{2}\right) x_{2} x_{3} .
\end{array}\right.
$$

Remark 1. Because the matrix $J_{24}$ satisfies the skew-symmetric structure, system $\Sigma_{24}$ is conservative both in the Hamiltonian and Casimir energy. The Casimir function, also known as energy momentum, is an important physical quantity in fluid dynamics. Its derivative is Casimir power, and in some cases, the Casimir power can be used as a criterion for determining whether a system can produce chaos. The system is conservative when $\dot{C} \equiv 0$ with $C \neq 0$. The Casimir function $C$ is properly selected as $C(\mathbf{x})=(1 / 2)\left(x_{1}^{2}+\right.$ $\left.x_{2}^{2}+x_{3}^{2}+x_{4}^{2}\right)$, which satisfies the Lie-Poisson brackets [35], $\{C, H\}=0, \forall H \in C^{\infty}\left(g^{*}\right)$.

According to the method from Reference [17], select functions $f_{3}(y, z)=h y, f_{4}(x, w)=a w, f_{5}(x, z)=e x$ and then obtain the constructed system

$$
\left\{\begin{array}{l}
\dot{x}=h y w \\
\dot{y}=a z w+e x w \\
\dot{z}=-a y w \\
\dot{w}=-(h+e) x y
\end{array}\right.
$$

Set $\Pi_{4}-\Pi_{2}=h, \Pi_{4}-\Pi_{3}=a, \Pi_{1}-\Pi_{4}=e, \Pi_{2}-\Pi_{1}=$ $-(h+e)$, and $\Pi_{3}-\Pi_{2}=0$; equation (5) can be transformed into the form of Euler equations (4). Because the constructed system (5) lacks a constant function, it cannot produce chaos, which corresponds to the Hamilton energy and 
Casimir energy conservation of four-dimensional Euler equations.

2.2. Case 1. The proposed system, equation (5), satisfies the four-dimensional Euler equation form. However, from Remark 1, this type of 4D Euler equations is conservative for Hamiltonian and Casimir energy, and it cannot generate chaos. So, we need to break the conservation. In $J_{24}^{H}(\mathbf{x}) \nabla H(\mathbf{x}), H(\mathbf{x})$ is Hamiltonian, and the vector field form is immutable. However, the matrix $J_{24}^{H}(\mathbf{x})$ is symplectic and can be transformed into skew-symmetric form. So, $J_{24}^{H}$ is obtained as

$$
J_{24}^{H}(\mathbf{x})=\left[\begin{array}{cccc}
0 & -x_{4} & c & x_{2} \\
x_{4} & 0 & -x_{4} & x_{3}-x_{1} \\
-c & x_{4} & 0 & -x_{2} \\
-x_{2} & x_{1}-x_{3} & x_{2} & 0
\end{array}\right]
$$

where $c$ is constant. And system $\Sigma_{24}^{H}$ is described as

$$
\Sigma_{24}^{H}\left\{\begin{array}{l}
\dot{x}_{1}=\left(\Pi_{4}-\Pi_{2}\right) x_{2} x_{4}+c \Pi_{3} x_{3}, \\
\dot{x}_{2}=\left(\Pi_{1}-\Pi_{4}\right) x_{1} x_{4}+\left(\Pi_{4}-\Pi_{3}\right) x_{3} x_{4}, \\
\dot{x}_{3}=\left(\Pi_{2}-\Pi_{4}\right) x_{2} x_{4}-c \Pi_{1} x_{1}, \\
\dot{x}_{4}=\left(\Pi_{2}-\Pi_{1}\right) x_{1} x_{2}+\left(\Pi_{3}-\Pi_{2}\right) x_{2} x_{3},
\end{array}\right.
$$

where $\Sigma_{24}^{H}$ represents the system that breaks the symplectic structure in $\Sigma_{24}$. System $\Sigma_{24}^{H}$ has 5 adjustable parameters: $\Pi_{i}, i=1,2,3,4$, and $c$, which can influence the orbital modes of system $\Sigma_{24}^{H}$, and they have physical meanings. The initial value, $x_{i 0}$, determines the total Hamiltonian; when the initial Hamiltonian is fixed, $\Pi_{i}$ is proportional to the frequency of the rigid body. For system $\Sigma_{24}^{H}$,

$$
\dot{H}(\mathbf{x})=\nabla H(\mathbf{x})^{T} J_{24}^{H}(\mathbf{x}) \nabla H(\mathbf{x})=0,
$$

and

$$
\dot{C}=\mathbf{x}^{T} \cdot \dot{\mathbf{x}}=\mathbf{x}^{T} \cdot J_{24}^{H}(\mathbf{x}) \cdot \nabla H(\mathbf{x})=c\left(\Pi_{3}-\Pi_{1}\right) x_{1} x_{3} .
$$

So, the Hamiltonian energy of system $\Sigma_{24}^{H}$ is conservative and the Casimir energy is nonconservative. In order to facilitate subsequent analysis, the parameter $c$ is reserved. Since the Euler equations satisfy that the sum of the coefficients of the coupling term is zero, it is calculated that $h, a$, and $e$ have a fixed relationship, that is, $a=h, e=-0.5 h$. And set $\Pi_{3}=d, \Pi_{1}=b$; then, the modified system $\Sigma_{24}^{H}$ can be transformed into our proposed equation form:

$$
\left\{\begin{array}{l}
\dot{x}=h y w+c \cdot d z, \\
\dot{y}=h z w-0.5 h x w, \\
\dot{z}=-h y w-c \cdot b x \\
\dot{w}=-0.5 h x y .
\end{array}\right.
$$

When $(h, d, b, c)=(-1,2.5,2,0.2)$ and the initial states $\left(x_{0}, y_{0}, z_{0}, w_{0}\right)=(0.5,0.5,1,1)$, system (10) is chaotic since its LEs are $(0.2405,0,0,-0.2405)$.
2.3. Case 2. Selecting function $f_{2}=g$, where $g$ is a constant, the constructed system is

$$
\left\{\begin{array}{l}
\dot{x}=h y w+g z, \\
\dot{y}=a z w+e x w \\
\dot{z}=-a y w-g x \\
\dot{w}=-(h+e) x y .
\end{array}\right.
$$

Set $(h, g, a, e)=(0.5,10,6,5.5)$ and $\left(x_{0}, y_{0}, z_{0}, w_{0}\right)=$ $(1,1,1,1)$; then, system (11) is chaotic since its LEs are $(1.0739,0,0,-1.0739)$.

Remark 2 illustrates that the Case 1 and Case 2 systems are fundamentally different.

Remark 2. Case 2 system can only be expressed as

$$
\left[\begin{array}{c}
\dot{x}_{1} \\
\dot{x}_{2} \\
\dot{x}_{3} \\
\dot{x}_{4}
\end{array}\right]=\left[\begin{array}{cccc}
0 & -x_{4} & 0 & x_{2} \\
x_{4} & 0 & -x_{4} & x_{3}-x_{1} \\
0 & x_{4} & 0 & -x_{2} \\
-x_{2} & x_{1}-x_{3} & x_{2} & 0
\end{array}\right]\left[\begin{array}{l}
\Pi_{1} x_{1} \\
\Pi_{2} x_{2} \\
\Pi_{3} x_{3} \\
\Pi_{4} x_{4}
\end{array}\right]+\left[\begin{array}{c}
g x_{1} \\
0 \\
g x_{3} \\
0
\end{array}\right] \text {. }
$$

This is because $\Pi_{3} \neq \Pi_{1}$; if we set $g=c \cdot \Pi_{3}$, it is clear that $g$ cannot be equal to $c \cdot \Pi_{1}$. But this form cannot get the Hamiltonian energy of Case 2 system. It should be expressed as follows:

$$
\left[\begin{array}{c}
\dot{x}_{1} \\
\dot{x}_{2} \\
\dot{x}_{3} \\
\dot{x}_{4}
\end{array}\right]=\left[\begin{array}{cccc}
0 & 0 & g & h x_{2} \\
0 & 0 & a x_{4} & e x_{1} \\
-g & -a x_{4} & 0 & 0 \\
-h x_{2} & -e x_{1} & 0 & 0
\end{array}\right]\left[\begin{array}{l}
x_{1} \\
x_{2} \\
x_{3} \\
x_{4}
\end{array}\right] \text {. }
$$

So, the Hamiltonian energy of Case 2 system is $H(\mathbf{x})=(1 / 2)\left(x_{1}^{2}+x_{2}^{2}+x_{3}^{2}+x_{4}^{2}\right)$.

\section{Equilibrium Point and Energy Analysis}

In this work, Matlab ODE45 algorithm is utilized to solve differential equations in the numerical simulations.

3.1. Equilibrium Point Analysis. Since the difference between these two systems is only the coefficients of the linear terms of the first and third equations, the coefficients do not affect the type and stability of the equilibrium point. Equilibrium points of Case 2 system are analyzed for simplicity. Let $\dot{x}=0, \dot{y}=0, \dot{z}=0$, and $\dot{w}=0$. The equilibrium point set of Case 2 is origin and two lines, and the two lines are the $y$-axis and the $w$-axis. Linearize $(11)$ at $S_{1}=(0,0,0,0)$, and the characteristic equation is

$$
\lambda^{2}\left(\lambda^{2}+100\right)=0 .
$$

Solve $\lambda_{1}=\lambda_{2}=0, \lambda_{3}=10 i, \lambda_{4}=-10 i$. So, equilibrium point $S_{1}$ is center. Linearize (11) at $S_{2}=\left(0, y^{*}, 0,0\right)$, and the characteristic equation is

$$
\lambda\left(\lambda^{3}+\left(100+3\left(y^{*}\right)^{2}\right) \lambda-360\left(y^{*}\right)^{2}\right)=0 .
$$


The eigenvalues $\lambda_{1}=0, \lambda_{2}=\sigma_{1}$, and $\lambda_{3,4}=-\sigma_{2} \pm j \omega_{1}$. Here, $\sigma_{1}, \sigma_{2}$, and $\omega_{1}$ are the functions of variable $y^{*}$. When $y^{*} \neq 0$, the eigenvalues are $\left(0, \sigma_{1},-\sigma_{2} \pm j \omega_{1}\right)$. Here, $\sigma_{1}=s-$ $m / s, \sigma_{2}=((m / 2 s)-(s / 2)), \omega_{1}=(\sqrt{3} / 2)((m / 2 s)+(s / 2)) i$, $m=\left(\left(y^{*}\right)^{2}+(100 / 3)\right)$, and $s=\left(\left(m^{3}+32400\left(y^{*}\right)^{4}\right)^{(1 / 2)}+\right.$ $\left.180\left(y^{*}\right)^{2}\right)^{(1 / 3)}$. So, equilibrium point $S_{2}$ is saddle foci.

Similarly, linearize (11) at $S_{3}=\left(0,0,0, w^{*}\right)$, and the characteristic equation is

$$
\lambda\left(\lambda^{3}+\left(100+33.25\left(w^{*}\right)^{2}\right) \lambda+360\left(w^{*}\right)\right)^{2}=0 .
$$

The eigenvalues $\lambda_{1}=0, \lambda_{2}=-\sigma_{3}$ and $\lambda_{3,4}=\sigma_{4} \pm j \omega_{2}$, where $\sigma_{3}, \sigma_{4}$, and $\omega_{2}$ are the functions of variable $w^{*}$. When $w^{*} \neq 0$, the eigenvalues are $\left(0,-\sigma_{3}, \sigma_{4} \pm j \omega_{2}\right)$, where $\sigma_{3}=$ $n-p / n, \sigma_{4}=((p / 2 n)-(n / 2)), \omega_{2}=(\sqrt{3} / 2)((p / 2 n)+(n / 2)) i$, $p=\left(\left(\left(133\left(w^{*}\right)^{2}\right) / 12\right)+(100 / 3)\right)$, and $n=\left(\left(32400\left(w^{*}\right)^{4}+\right.\right.$ $\left.\left(p^{3}\right)^{(1 / 2)}-180\left(w^{*}\right)^{2}\right)^{(1 / 3)}$.

Finally, the equilibrium points of Case 1 system and Case 2 system are summarized in Table 1 . The systems are Hamiltonian conservative; therefore, these two systems cannot arrive at an equilibrium point asymptotically. This implies the nodes and spirals cannot exist in the conservative system, while the elliptic (center) and hyperbolic (saddle) equilibrium points can occur.

\subsection{The Mechanism of Chaos and Dynamic Analysis}

3.2.1. Case 1. The mechanism analysis of the Casimir power is an effective method in the study of the generation process of chaos. Casimir energy is conservative when no external or dissipative forces are included in systems. For Case 1 system, the Casimir energy is not conservative, which means that there must be an external torque or a dissipative torque. Arnor'd [25] put forward a Kolmogorov system in a 3D form, which described the dissipative-forced dynamical systems or the hydrodynamic instability:

$$
\dot{x}=\{x, H\}-\Lambda x+f,
$$

where $x=\left\{x_{1}, x_{2}, x_{3}\right\}^{T},\{$,$\} is the Lie-Poisson structure, and$ $\Lambda x$ and $f$ represent the dissipative and external forces, respectively. The Kolmogorov form is useful for studying energy cycle in systems. In general, $\{x, H\}$ are quadratic coupling terms, and $\Lambda x$ is a linear term. Use this idea to separate the conservative and dissipative parts of system $\Sigma_{24}^{H}$. To illustrate this more clearly, equation (4) is rewritten as

$$
\left[\begin{array}{c}
\dot{x}_{1} \\
\dot{x}_{2} \\
\dot{x}_{3} \\
\dot{x}_{4}
\end{array}\right]=\left[\begin{array}{c}
\left(\Pi_{4}-\Pi_{2}\right) x_{2} x_{4} \\
\left(\Pi_{1}-\Pi_{4}\right) x_{1} x_{4}+\left(\Pi_{4}-\Pi_{3}\right) x_{3} x_{4} \\
\left(\Pi_{2}-\Pi_{4}\right) x_{2} x_{4} \\
\left(\Pi_{2}-\Pi_{1}\right) x_{1} x_{2}+\left(\Pi_{3}-\Pi_{2}\right) x_{2} x_{3}
\end{array}\right]+\left[\begin{array}{c}
c \Pi_{3} x_{3} \\
0 \\
-c \Pi_{1} x_{1} \\
0
\end{array}\right] .
$$

From Remark 1, it can be found that the term $J_{24}(\mathbf{x}) \nabla H(\mathbf{x})$ is conservative in both Hamiltonian and Casimir functions. Therefore, this term is an inertial torque, which corresponds to the first vector term to the right of the equal sign in equation (18). It is the fictitious torque generated by a free rotational rigid body with no external torque or dissipative torque. However, the term

$$
J^{H}(\mathbf{x}) \nabla H_{1}(\mathbf{x})=\left[c \Pi_{3} x_{3} 0-c \Pi_{1} x_{1} 0\right]^{T},
$$

is a nonconservative torque, which corresponds to the second part of equation (18). And this term is the external torque, which induces the energy exchanges between dissipative and supplied energy, thereby bringing about the oscillation of the Casimir power.

It is worth noting that the external torque in equation (18) actually includes the dissipative torque. Since the sign of $c \Pi_{3} x_{3}$ and $-c \prod_{1} x_{1}$ is uncertain, it is impossible to determine whether it is dissipative or external torque. There are two terms in the total Casimir power $\dot{C}=c\left(\Pi_{3}-\Pi_{1}\right) x_{1} x_{3}$ : (1) $c \Pi_{3} x_{1} x_{3}$ (the upper curve in Figure 1(a)), in which the power is supplied by the external torque $c \Pi_{3} x_{3}$, and (2) $-c \Pi_{1} x_{1} x_{3}$ (the lower curve in Figure 1(a)), in which the power is supplied by the external torque $-c \Pi_{1} x_{1}$. The term, which is greater (less) than zero, takes a supply (dissipation) role. It was concluded that both of them were responsible for dissipative or external torque.

Equations (8) and (9) are also verified by the numerical simulation results. The green line in Figure 1(b) represents the Hamiltonian energy of Case 1, which does not change over time and remains at 2.625. The irregular oscillating blue curve in Figure 1(b) is the Casimir energy. Chaotic orbits displaying Hamiltonian energy and Casimir energy are shown in Figures 2(a) and 2(b), respectively.

The initial value is $(0.5,0.5,1,1)$ and parameters are $(h, d, b)=(-1,2.5,2)$. When $c=0$, Case 1 system is the $4 \mathrm{D}$ Euler equation $\Sigma_{24}$; therefore, it is conservative in both the Hamiltonian with $H(t)=2.5625$ and Casimir energy with $C(t)=1.25$. The system produces periodic states (red curve in Figure 3(a)), the Casimir power is on the zero line (red curve in Figure $3(\mathrm{~b})$ ). If we set $c=0.2$, the Hamiltonian is still conservative with $H(t)=2.5625$, but it oscillates irregularly in the Casimir power (blue curve in Figure 3(b)), and the original periodic orbit is perturbed and turns into chaotic (blue curve in Figure 3(a)). Although the excitation is small as far as the value of $c$ is concerned, the response of the system has essentially changed because the system has completely changed its orbital mode from regular to chaotic, as verified by the Lyapunov exponents $\mathrm{LE}=[0.2405$, $0,0,-0.2405]$. The red curves in Figures 4(a) and 4(b) are the periodic orbits in 3D space of $x_{1}-x_{2}-x_{4}$ and the phase portrait of $x_{2}-x_{4}$ produced by Case 1 without the excitation and the blue curves are the chaotic orbits produced by Case 1 under excitation, respectively.

Bifurcation diagram and Lyapunov exponent spectrum of Case 1 system are shown in Figures 5(a) and 5(b). Normally, the bifurcation transition occurs from source to periodic orbit, double periodic orbits, and multiperiodic orbits until chaos or an inverse evolution of dynamical bifurcation [36]. However, Case 1 system does not undergo such a bifurcation transition. It enters the chaotic mode suddenly as long as parameter $c>0$, as shown in the bifurcation diagram, where $c$ varies in the interval $c \in[0,0.2]$. 
Table 1: Equilibrium point type of Case 1 and Case 2 systems.

\begin{tabular}{|c|c|c|c|c|}
\hline System & System model & Equilibrium point $\left(y^{*}, w^{*} \in R\right)$ & Eigenvalue $\left(\sigma, \omega \in R^{+}\right)$ & Equilibrium type \\
\hline Case 1 & $\begin{array}{l}\dot{x}=h y w+c \cdot d z \\
\dot{y}=h z w-0.5 h x w \\
\dot{z}=-h y w-c \cdot b x \\
\dot{w}=-0.5 h x y\end{array}$ & $\begin{array}{l}(0,0,0,0) \\
\quad \text { or } \\
\left(0, y^{*}, 0,0\right) \\
\quad \text { or } \\
\left(0,0,0, w^{*}\right)\end{array}$ & $\begin{array}{c}(0,0, \pm 0.5 j) \\
\text { or } \\
\left(0, \sigma_{5},-\sigma_{6} \pm j \omega_{3}\right) \\
\text { or } \\
\left(0,-\sigma_{7}, \sigma_{8} \pm j \omega_{4}\right)\end{array}$ & $\begin{array}{c}\text { Center } \\
\text { Saddle - foci } \\
\text { Saddle - foci }\end{array}$ \\
\hline Case 2 & $\left\{\begin{array}{l}\dot{x}=h y w+g z \\
\dot{y}=a z w+e x w \\
\dot{z}=-a y w-g x \\
\dot{w}=-(h+e) x y\end{array}\right.$ & $\begin{array}{l}(0,0,0,0) \\
\quad \text { or } \\
\left(0, y^{*}, 0,0\right) \\
\quad \text { or } \\
\left(0,0,0, w^{*}\right)\end{array}$ & $\begin{array}{c}(0,0, \pm 10 j) \\
\text { or } \\
\left(0, \sigma_{1},-\sigma_{2} \pm j \omega_{1}\right) \\
\quad \text { or } \\
\left(0,-\sigma_{3}, \sigma_{4} \pm j \omega_{2}\right)\end{array}$ & $\begin{array}{c}\text { Center } \\
\text { Saddle - foci } \\
\text { Saddle - foci }\end{array}$ \\
\hline
\end{tabular}

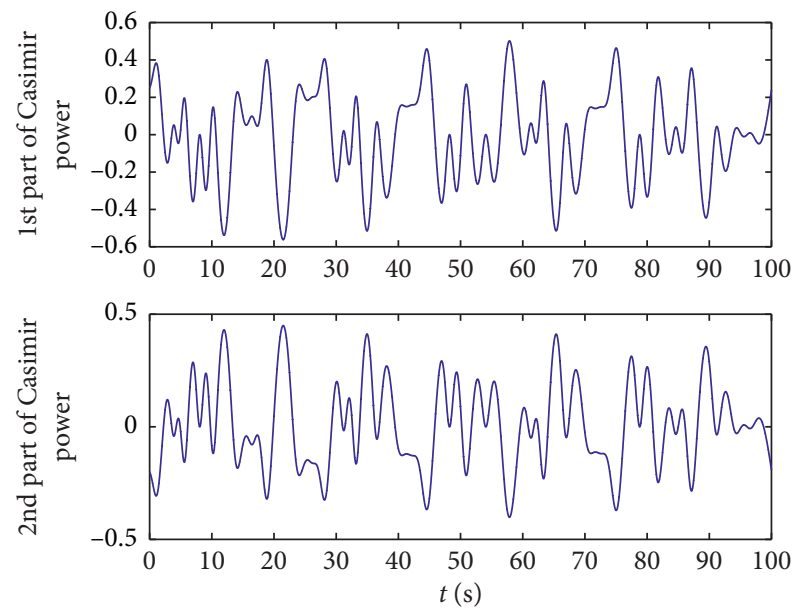

(a)

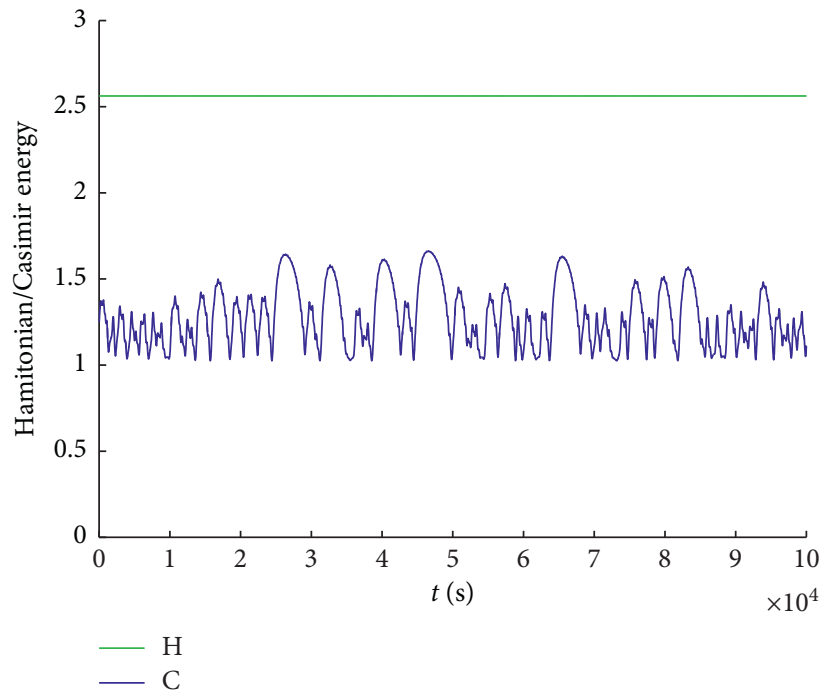

(b)

Figure 1: Casimir power, Hamilton energy, and Casimir energy of Case 1 system. (a) Two parts of Casimir powers. (b) Hamilton energy and Casimir energy.

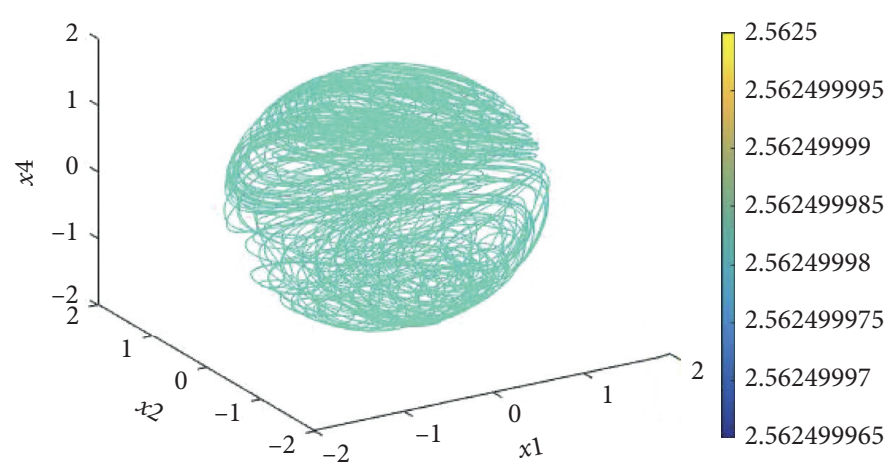

(a)

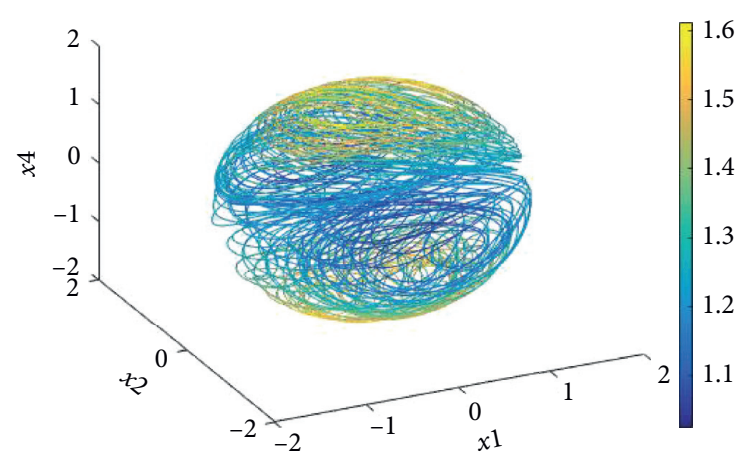

(b)

Figure 2: Chaotic orbits of Case 1 system. (a) Hamilton energy. (b) Casimir energy. 


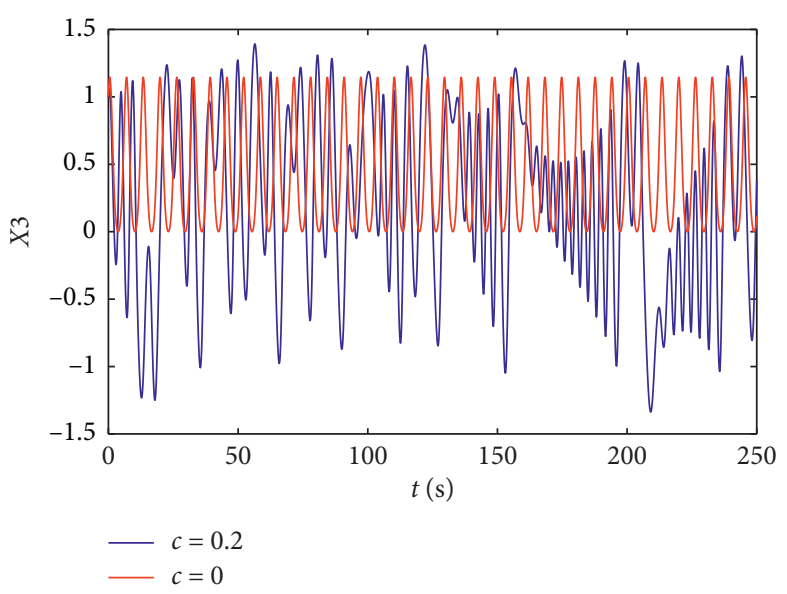

(a)

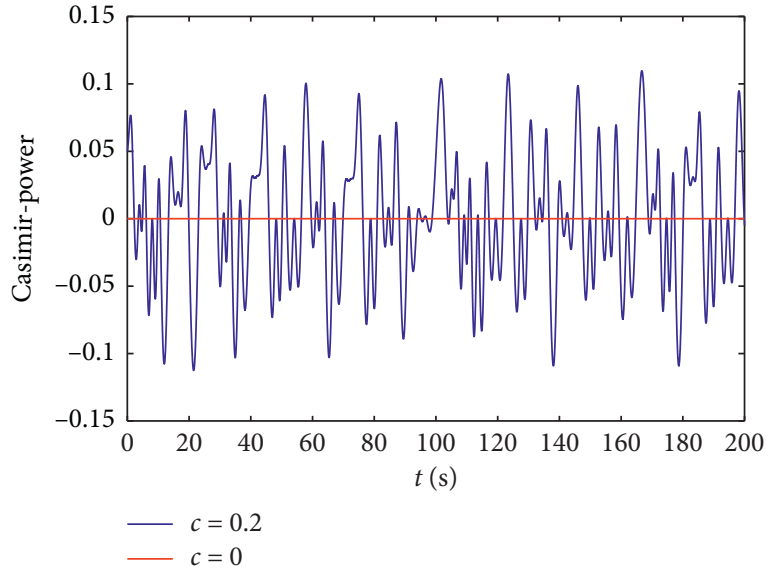

(b)

Figure 3: Time series of $x_{3}$ and Casimir powers of Case 1 system, with $(h, d, b)=(-1,2.5,2)$ and $\left(x_{0}, y_{0}, z_{0}, w_{0}\right)=(0.5,0.5,1,1)$. (a) Time series of $x_{3}$. (b) Casimir powers.

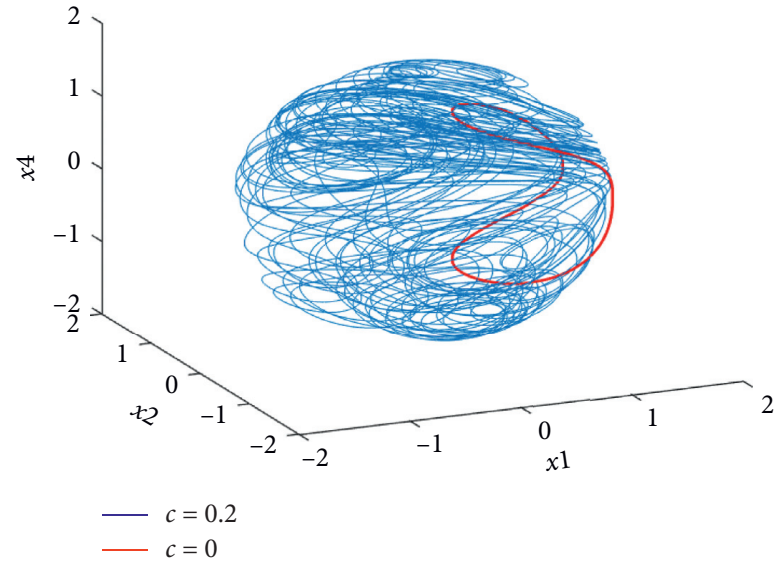

(a)

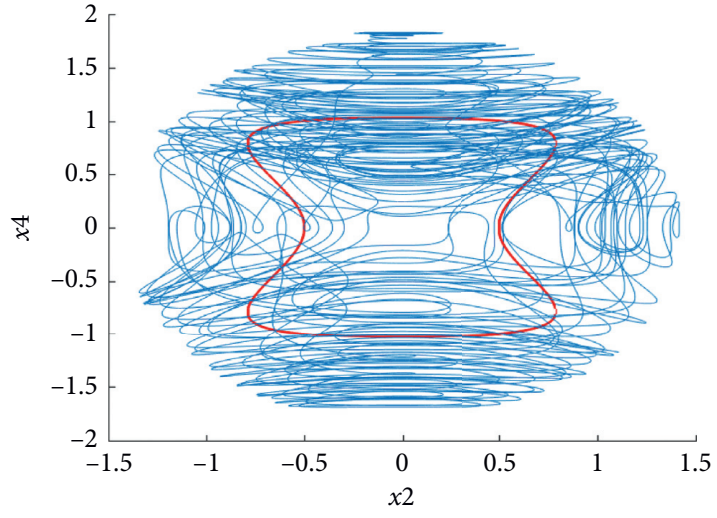

$-c=0.2$

(b)

Figure 4: Phase portraits of Case 1 system on the different plane, with $(h, d, b)=(-1,2.5,2)$ and $\left(x_{0}, y_{0}, z_{0}, w_{0}\right)=(0.5,0.5,1,1)$. (a) 3D space of $x_{1}-x_{2}-x_{4}$. (b) $x_{2}-x_{4}$ plane

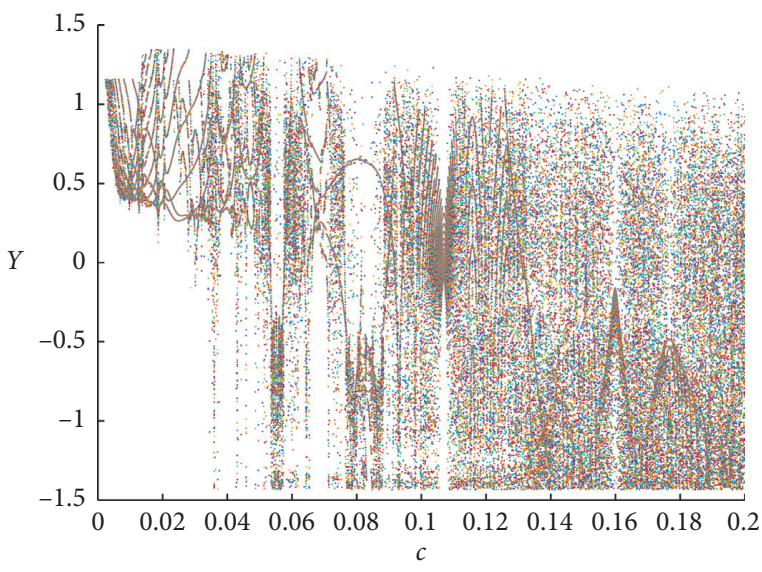

(a)

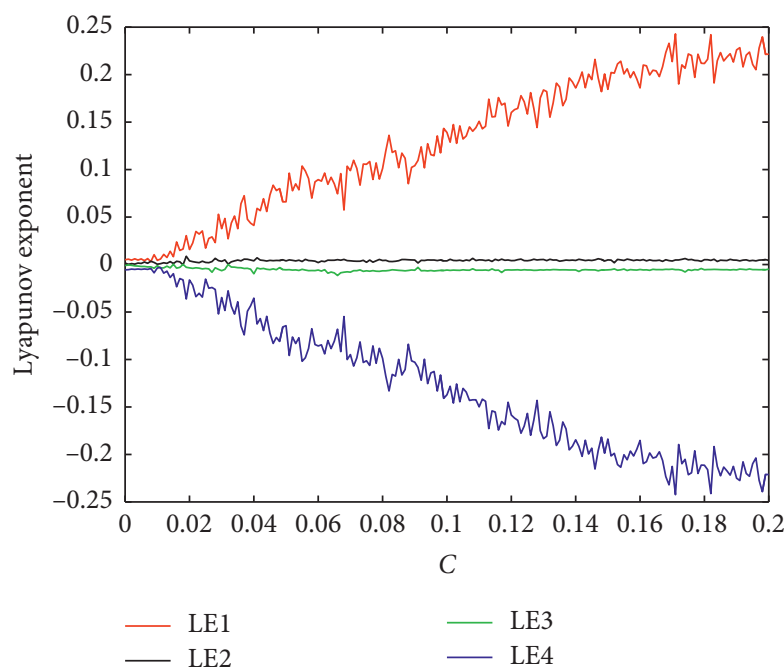

(b)

FIGURE 5: Lyapunov exponent spectrum and bifurcation diagram of Case 1 system versus $c$. (a) Bifurcation diagram. (b) Lyapunov exponent spectrum. 
3.2.2. Case 2. But there is an interesting phenomenon during the mechanism analysis of Case 2 system.

In addition to the invariants such as energy and momentum that are associated with explicit symmetries, the noncanonical Hamiltonian systems generally possess Casimir invariants or distinguished functions. The canonical form is represented as

$$
J=\left[\begin{array}{cc}
0 & E \\
-E & 0
\end{array}\right],
$$

where $E$ is an $n \times n$ identity matrix and 0 is an $n \times n$ zero matrix. From Remark 2,

$$
\begin{aligned}
\dot{x} & =J(\mathbf{x}) \cdot \nabla H(\mathbf{x}) \\
& =\left[\begin{array}{cccc}
0 & 0 & g & h x_{2} \\
0 & 0 & a x_{4} & e x_{1} \\
-g & -a x_{4} & 0 & 0 \\
-h x_{2} & -e x_{1} & 0 & 0
\end{array}\right]\left[\begin{array}{l}
x_{1} \\
x_{2} \\
x_{3} \\
x_{4}
\end{array}\right] .
\end{aligned}
$$

It is obvious that the structure matrix of Case 2 system is canonical, and a canonical system has no nontrivial Casimirsfunctions [33].

Since Casimir power cannot explain the chaos generation mechanism of Case 2 system, we have to find another way. Kokubu and Roussarie verified the existence of one specific heteroclinic cycle, called the singularly degenerate heteroclinic cycle, which consists of an invariant set formed by a line of equilibria together with a heteroclinic orbit connecting two of the equilibria [37]. They also found that the rupture of this cycle would separate the chaotic attractors. Are there such orbits in Case 2 system? In this part, by choosing the appropriate parameters and performing a large number of Matlab numerical simulations, the cycle was discovered. According to Section 3.1, the system has nonisolated equilibrium points $E_{y}$ and $E_{w}$. The characteristic equation of $E_{y}$ is

$$
\lambda\left(\lambda^{3}+\left(h(h+e)\left(y^{*}\right)^{2}+g^{2}\right) \lambda-g \cdot a \cdot(h+e)\left(y^{*}\right)^{2}\right)=0,
$$

where $\tau_{1}=0, \quad \tau_{2}=h(h+e)\left(y^{*}\right)^{2}+g^{2}$, and $\tau_{3}=-g \cdot a$. $(h+e)\left(y^{*}\right)^{2}$, and it is easy to know that each $E_{y}$ is hyperbolic. The characteristic equation of $E_{w}$ is

$$
\lambda\left(\lambda^{3}+\left(\left(a^{2}-h \cdot e\right)\left(w^{*}\right)^{2}+g^{2}\right) \lambda+g \cdot a \cdot(h+e)\left(w^{*}\right)^{2}\right)=0,
$$

where $\rho_{1}=0, \rho_{2}=\left(a^{2}-h \cdot e\right)\left(w^{*}\right)^{2}+g^{2}$, and $\rho_{3}=g \cdot a$. $(h+e)\left(w^{*}\right)^{2}$, and it is easy to know that each $E_{w}$ is hyperbolic. According to the Routh-Hurwitz criterion, Remark 3 is established.

Remark 3. If parameters $a, h, e, g$, and $y^{*}\left(w^{*}\right)$ satisfy $\left.\tau_{1}>0\left(\rho_{1}>0\right), \quad \tau_{2}>0\left(\rho_{2}>0\right)\right), \quad \tau_{3}>0\left(\rho_{3}>0\right)$, and $\tau_{1} \tau_{2}-$ $\tau_{3}>0\left(\rho_{1} \rho_{2}-\rho_{3}>0\right)$, then $E_{y}\left(E_{w}\right)$ is a stable node-like or focus-like equilibrium point. Otherwise, $E_{y}\left(E_{w}\right)$ is unstable or more degraded.

Take $E_{y}$ as an example. Selecting appropriate parameter values and initial values, a large number of Matlab numerical simulations were carried out, and the following conclusions are obtained. If parameters $a, h, e, g$, and $y^{*}$ do not satisfy $\tau_{1}>0, \tau_{2}>0, \tau_{3}>0$, and $\tau_{1} \tau_{2}-\tau_{3}>0$, then the $1 \mathrm{D}$ (or 2D) unstable manifold $W^{u}\left(E_{y_{1}}\right)$ of each saddle point or unstable focus-like $E_{y_{1}}=\left(0, y_{1}, 0,0\right)$ will tend to normal hyperbolic stable manifold $W^{s}\left(E_{y_{2}}\right)$ of a stable focus $E_{y_{2}}=\left(0, y_{2}, 0,0\right)$, as shown in Figure 6 . Case 2 system has infinite singularly degenerate heteroclinic cycles, and Figure 7 shows that chaotic attractors are generated near the singularly degenerate heteroclinic cycle.

For Case 2 system, the phase trajectory and Poincare section are drawn in Figures 8-11 to verify the dynamic behaviors under different parameters. The bifurcation diagram (Figure 12(a)) and Lyapunov exponent spectrum (Figure 12(b)) shows that when $g \in(0,16)$, Case 2 system generates a chaotic flow and when $g \in[16,25)$, Case 2 system enters into quasiperiodic motion. It can be seen from the phase trajectory (Figure 8) and the Poincare section (Figure 9) that when $g=10$, the system is chaotic. If $g$ is 20 , the system shows a quasiperiodic state (Figures 10 and 11). It is found that the parameters $h$ and $g$ have great impact on the dynamic behaviors of Case 2 system through many simulations. If we fix the initial values with $\left(x_{0}, y_{0}, z_{0}, w_{0}\right)=(1,1,1,1)$ but change the parameters, the maps of four Lyapunov exponents related to parameters $h$ and $g$ can be obtained as shown in Figures 12(a) and 12(b), and the LEs with different values are highlighted with different colors. It can be seen from Figure 13 that LE1 and LE4 are symmetrical and LE2 and LE3 are symmetrical, which is also consistent with the characteristics of the Lyapunov exponent of the conservative system $[38,39]$.

\section{National Institute of Standards and Technology (NIST) Test and Field Programmable Gate Array (FPGA) Implementation}

4.1. NIST Test for Two Cases. The proposed systems can be used to make a random or pseudorandom number generator. National Institute of Standards and Technology [40] provides the SP800-22 standard, which is a very common method that can be used to evaluate pseudorandom sequences identified in the field of encryption. It contains 15 tests. The number of pseudorandom sequences tested in Case 1 and Case 2 is 95, and the length of each group is 100 million bits. The test results of these two systems are listed in Tables 2 and 3, which contain the $P$ - value of each NIST test, the distribution of the $P$ - values, and the pass proportion of the 95 groups. For these 15 tests, each $P$ - value is the probability that a perfect random number generator would have produced a sequence less random than the tested sequence. A significance level " $\alpha$ " is chosen for the tests, which represents the probability of a Type-I error, and its range is from 0.001 to 0.01 . If $P$ - value $\geq \alpha$, then the null hypothesis is accepted, i.e., the sequence appears to be random. If $P-$ value $<\alpha$, the sequence is nonrandom due to the rejected null hypothesis.

According to the SP800-22 standard [40], the test results have to satisfy three conditions: 


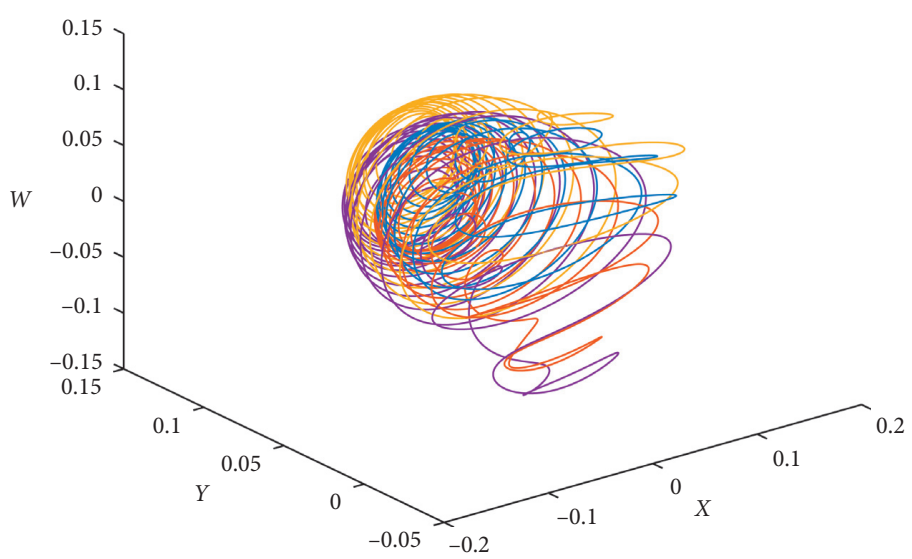

(a)

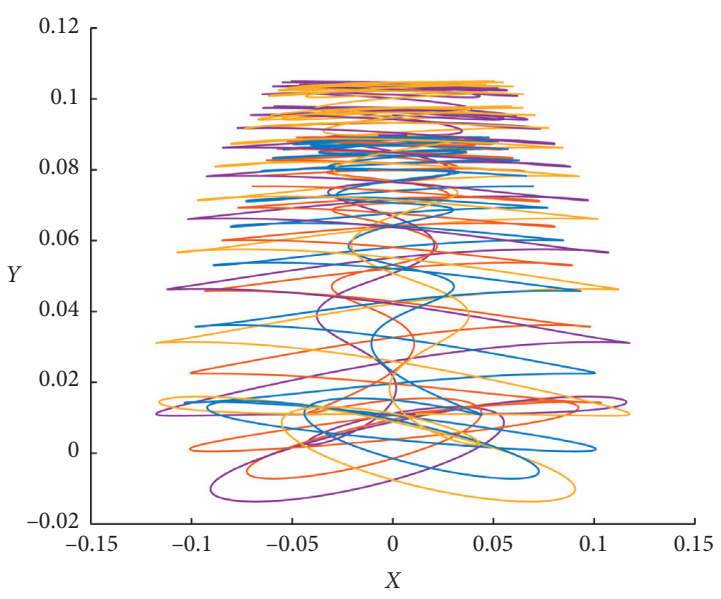

(b)

Figure 6: Singularly degenerate heteroclinic cycles of Case 2 system: set $(h, a, e, g)=(-25,-2,1,0.7)$ and $\left(x_{0}, y_{0}, z_{0}, w_{0}\right)=\left( \pm 0.0918, y^{*}, \pm 0.00469, \pm 0.00290\right)$, where $y^{*}=0.05,0.08$. (a) $x-y-w$. (b) $x-y$.

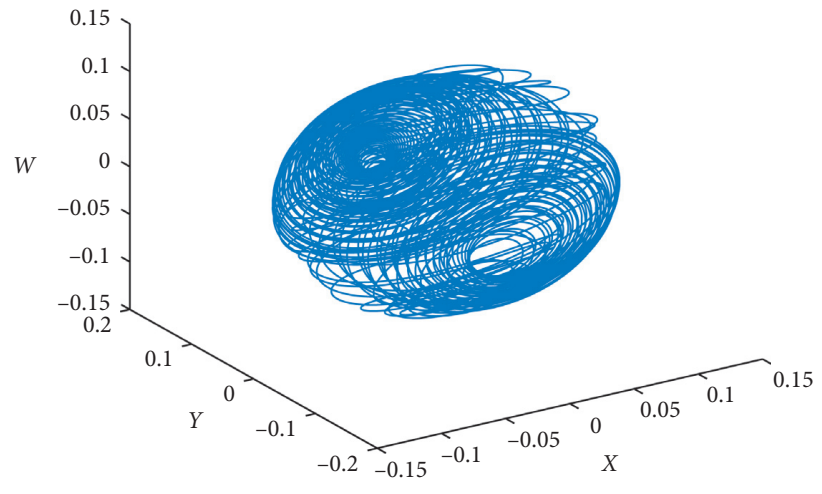

(a)

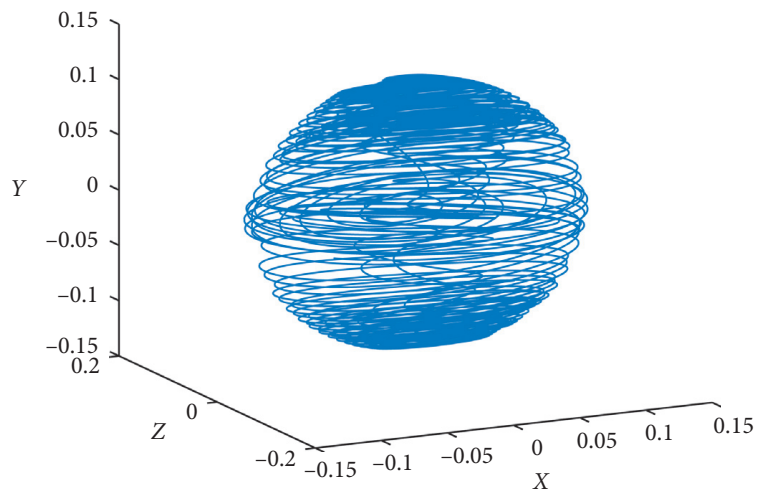

(b)

Figure 7: Phase portraits of Case 2 system: set $(h, a, e, g)=(-25,-2,1,0.7),(a)\left(x_{0}, y_{0}, z_{0}, w_{0}\right)=(0.0918,0.08,0.00469,0.00290)$, and (b) $\left(x_{0}, y_{0}, z_{0}, w_{0}\right)=(-0.0918,0.08,-0.00469,-0.00290)$.

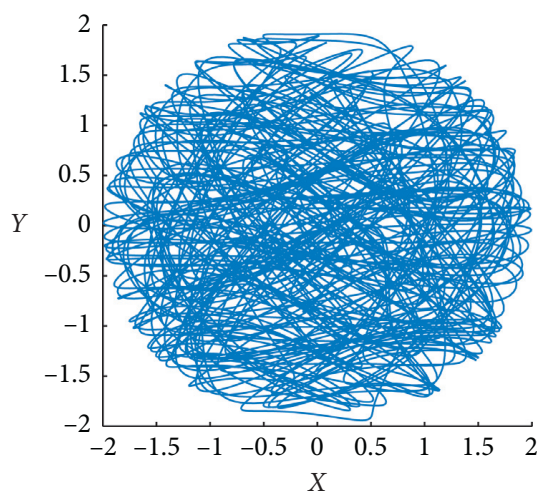

(a)

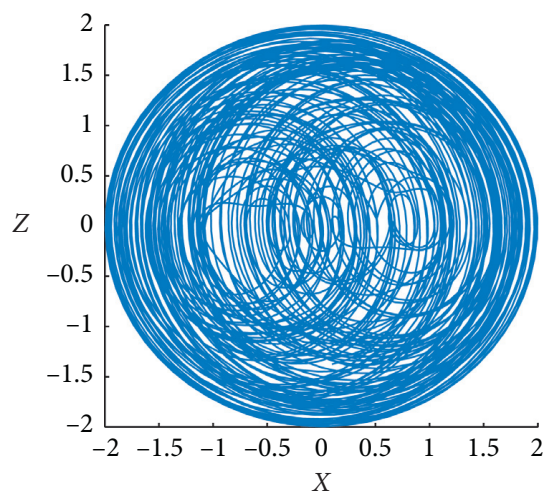

(b)

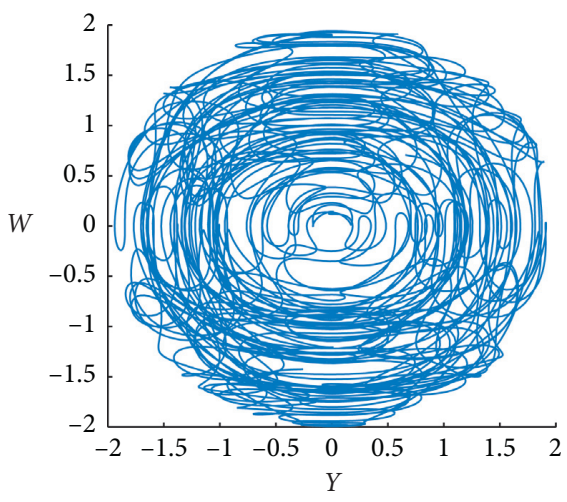

(c)

FIgURE 8: Phase portraits of Case 2 system on the different plane, with $(h, g, a, e)=(0.5,10,6,5.5)$ and $\left(x_{0}, y_{0}, z_{0}, w_{0}\right)=(1,1,1,1)$. (a) $x-y$ plane. (b) $x-z$ plane. (c) $y$-w plane. 


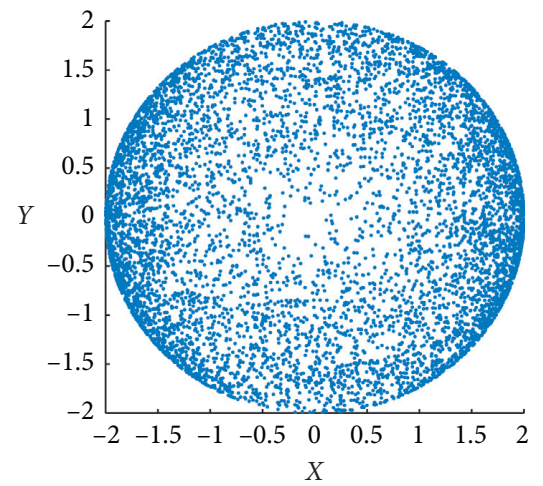

(a)

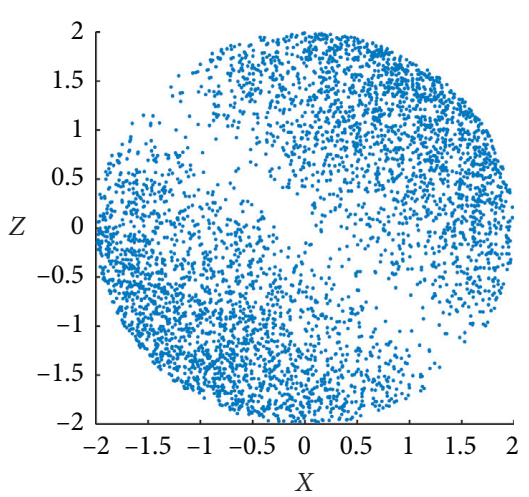

(b)

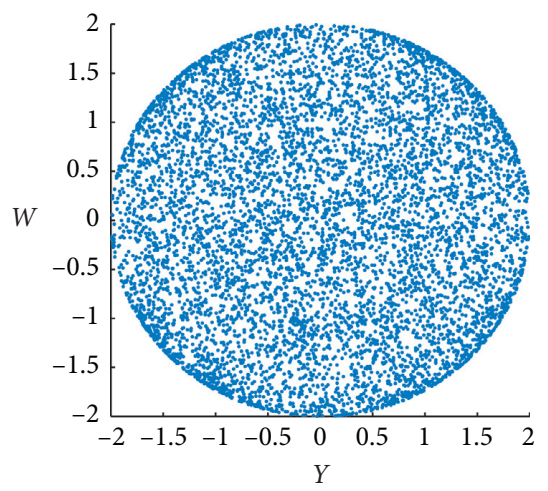

(c)

Figure 9: Phase portraits of Case 2 system on the different plane, with $(h, g, a, e)=(0.5,10,6,5.5)$ and $\left(x_{0}, y_{0}, z_{0}, w_{0}\right)=(1,1,1,1)$. (a) $x-y$ plane. (b) $x-z$ plane. (c) $y$-w plane.

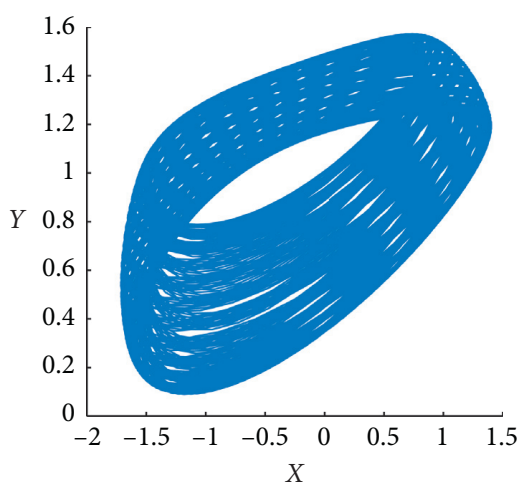

(a)

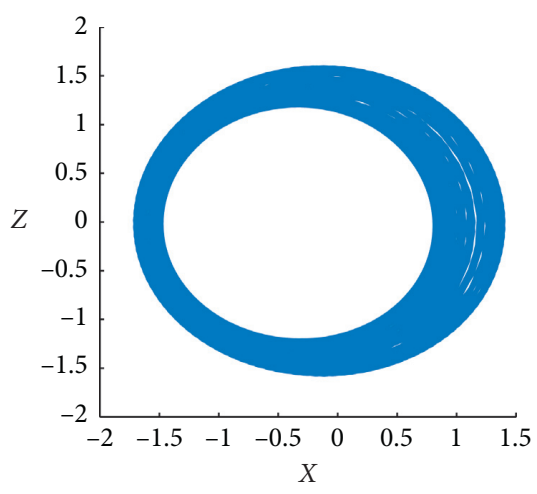

(b)

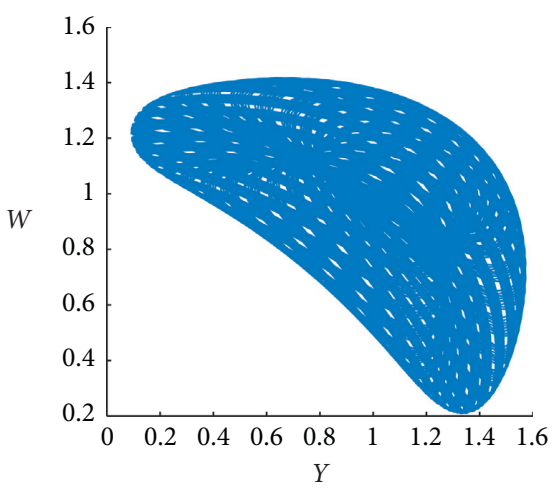

(c)

Figure 10: Phase portraits Poincare sections of Case 2 system on the different plane, with $(h, g, a, e)=(0.5,20,6,5.5)$ and $\left(x_{0}, y_{0}, z_{0}, w_{0}\right)=(1,1,1,1)$. (a) $x$-y plane. (b) $x$ - $z$ plane. (c) $y$-w plane.

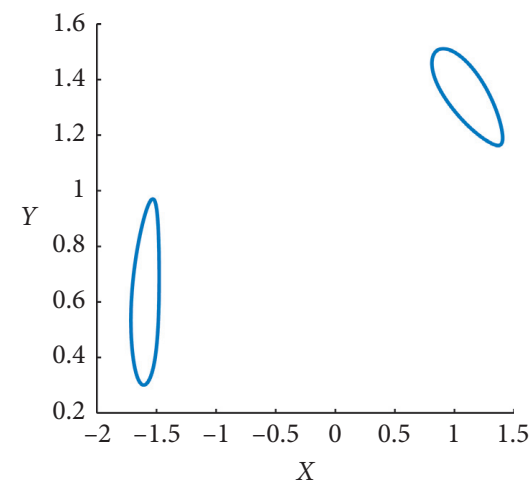

(a)

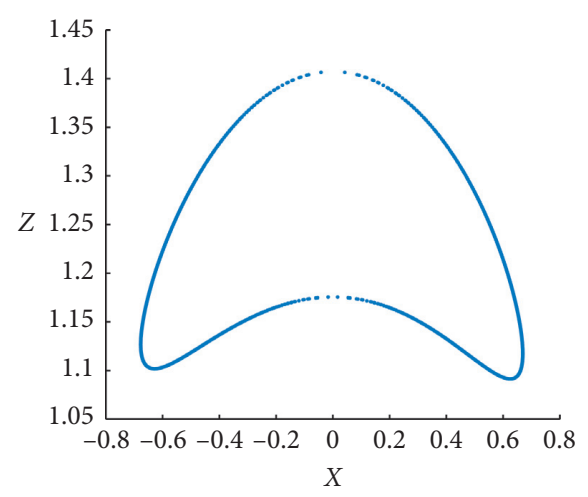

(b)

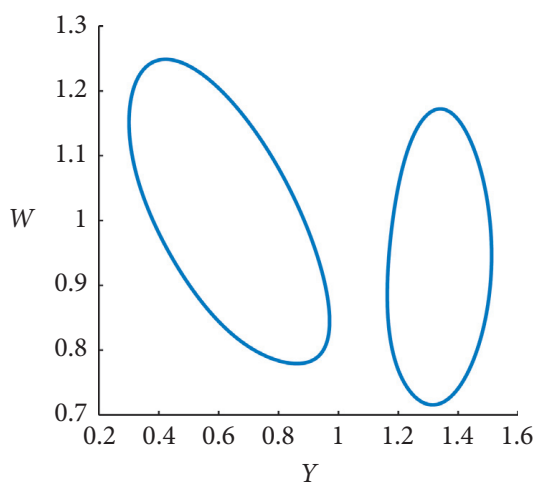

(c)

Figure 11: Poincare sections of Case 2 system on the different plane, with $(h, g, a, e)=(0.5,20,6,5.5)$ and $\left(x_{0}, y_{0}, z_{0}, w_{0}\right)=(1,1,1,1)$. (a) $x-y$ plane. (b) $x-z$ plane. (c) $y-w$ plane. 


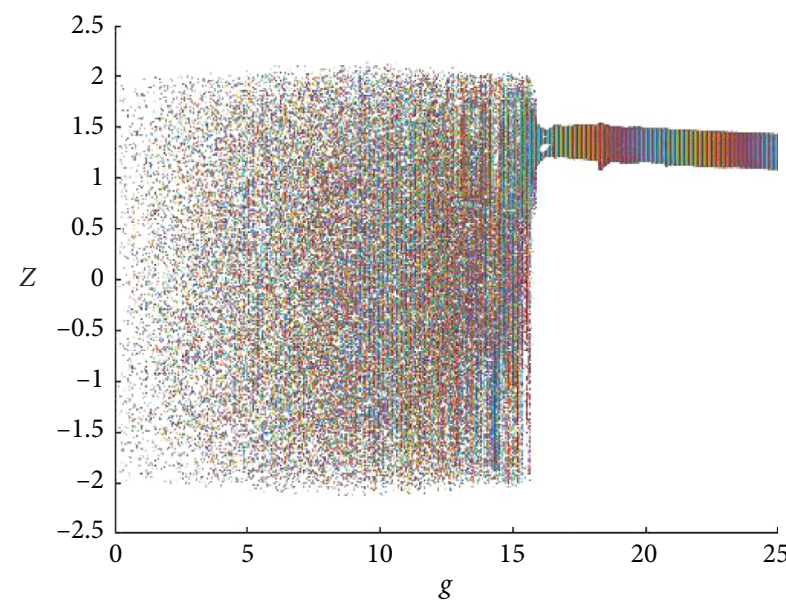

(a)

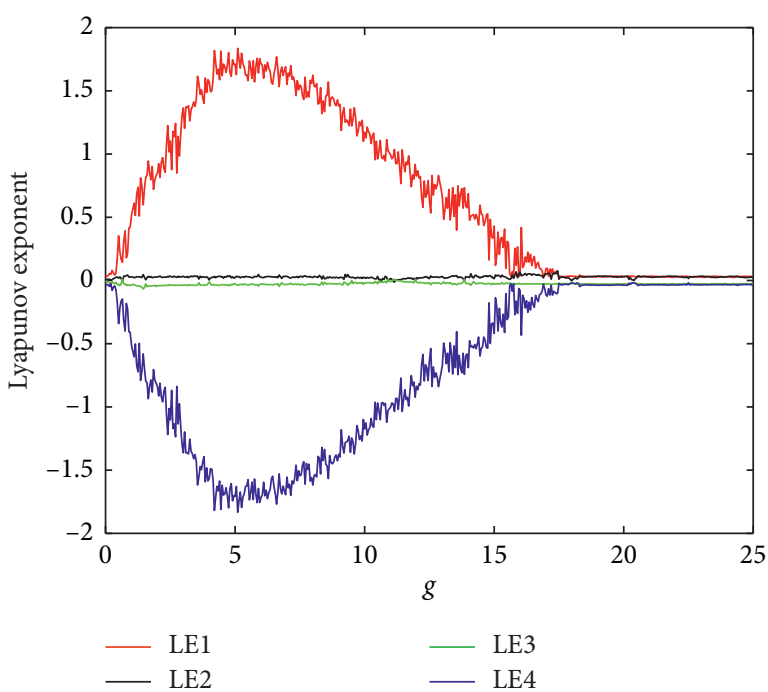

(b)

FiguRe 12: Lyapunov exponent spectrum and bifurcation diagram of Case 2 system versus g. (a) Bifurcation diagram. (b) Lyapunov exponent spectrum.

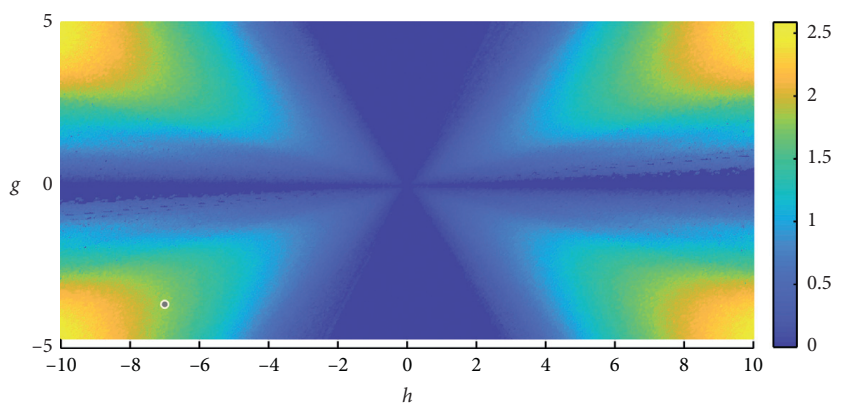

(a)

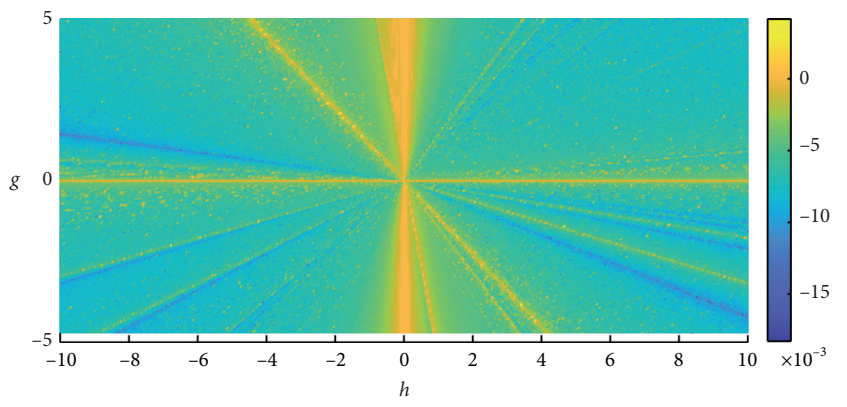

(c)

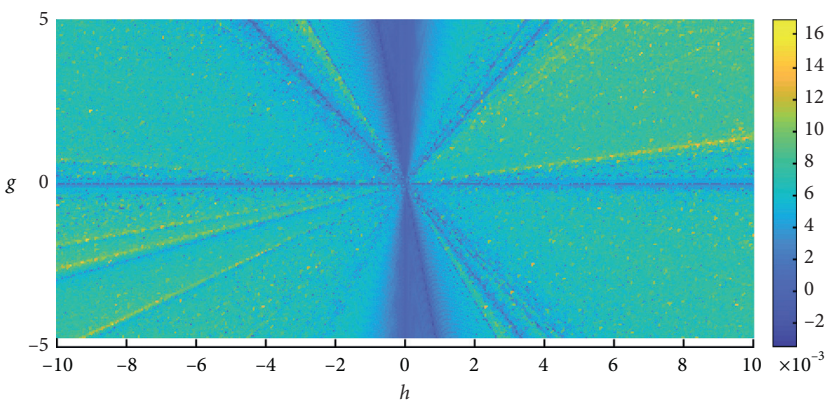

(b)

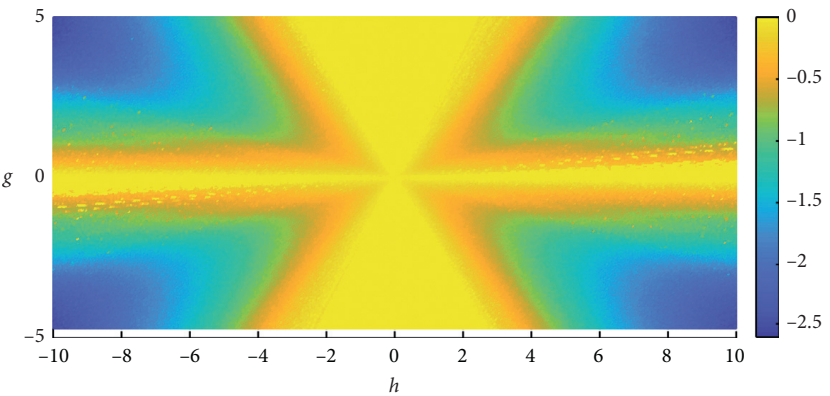

(d)

Figure 13: Dynamical evolution map of Case 2 system with $h \in(-10,10), g \in(-5,5)$. (a) LE1. (b) LE2. (c) LE3. (d) LE4.

(a) All $P$ - values must be greater than the significance level, $\alpha=0.01$.

(b) A proportion must pass the confidence interval formulated as

$$
\widehat{p} \pm 3 \sqrt{\frac{\widehat{p}(1-\widehat{p})}{m}},
$$

where $\widehat{p}=1-\alpha, m=95$, implying the proportion should be between 0.9601 and 1.0298 . (c) Since the $P$ values of the straight random sequence obey uniform distribution, the randomness can be described according to the uniformity of the distribution of $P$-values. The $P$-value is a number between 0 and 1 , so we divide 0 to 1 into 10 intervals: $[0.0,0.1),[0.1,0.2),[0.2,0.3),[0.3,0.4),[0.4,0.5)$, $[0.5,0.6), \quad[0.6,0.7), \quad[0.7,0.8), \quad[0.8,0.9)$, and $[0.9,1.0]$. The value of $\mathrm{C} 1-\mathrm{C} 10$ is the number of these $10 P$-values in the corresponding interval. Since the data are divided into 95 groups, the sum of 
TABLE 2: NIST test result of Case 1 system.

\begin{tabular}{lcccccccccccccc}
\hline$C 1$ & $C 2$ & $C 3$ & $C 4$ & $C 5$ & $C 6$ & $C 7$ & $C 8$ & $C 9$ & $C 10$ & $P$ value & $P$ value & Proportion & Statistical test \\
\hline 11 & 8 & 9 & 11 & 11 & 9 & 10 & 7 & 12 & 7 & 0.9440 & 0.9707 & 0.989 & Frequency \\
12 & 12 & 9 & 8 & 7 & 3 & 10 & 9 & 7 & 18 & 0.0646 & 0.1225 & 0.989 & Block frequency \\
10 & 10 & 14 & 10 & 9 & 14 & 7 & 4 & 9 & 8 & 0.104170 & 0.6130 & 0.979 & Cumulative sums \\
9 & 11 & 6 & 16 & 7 & 11 & 6 & 8 & 12 & 9 & 0.3595 & 0.5526 & 1 & Runs \\
7 & 13 & 4 & 9 & 12 & 7 & 11 & 13 & 10 & 9 & 0.4580 & 0.6528 & 1 & Longest run \\
14 & 6 & 7 & 11 & 9 & 9 & 8 & 16 & 7 & 8 & 0.2912 & 0.4728 & 0.968 & Rank \\
14 & 14 & 6 & 5 & 11 & 7 & 10 & 10 & 6 & 12 & 0.2464 & 0.4150 & 0.968 & FFT \\
11 & 9 & 21 & 5 & 8 & 6 & 9 & 6 & 9 & 11 & 0.0137 & 0.0221 & 1 & Nonoverlapping template \\
5 & 17 & 8 & 10 & 12 & 15 & 4 & 6 & 10 & 8 & 0.0339 & 0.0622 & 0.989 & Overlapping template \\
13 & 11 & 13 & 10 & 12 & 4 & 5 & 7 & 11 & 9 & 0.3074 & 0.4925 & 0.989 & Universal \\
9 & 7 & 11 & 11 & 7 & 14 & 7 & 8 & 11 & 10 & 0.7728 & 0.8849 & 0.989 & Approximate entropy \\
3 & 4 & 8 & 6 & 2 & 12 & 2 & 8 & 6 & 4 & 0.0288 & 0.0734 & 1 & Random excursions \\
2 & 11 & 4 & 2 & 6 & 7 & 5 & 6 & 3 & 9 & 0.0628 & 0.1552 & 0.982 & Random excursion variant \\
10 & 10 & 7 & 16 & 10 & 8 & 11 & 7 & 3 & 13 & 0.1626 & 0.2931 & 1 & Serial \\
8 & 11 & 6 & 8 & 13 & 9 & 12 & 5 & 13 & 10 & 0.5229 & 0.7106 & 1 & Linear complexity \\
\hline
\end{tabular}

TABLE 3: NIST test result of Case 2 system.

\begin{tabular}{lccccccccccccc}
\hline$C 1$ & $C 2$ & $C 3$ & $C 4$ & $C 5$ & $C 6$ & $C 7$ & $C 8$ & $C 9$ & $C 10$ & $P$-value & $P$-value $T$ & Proportion & Statistical test \\
\hline 16 & 12 & 10 & 8 & 9 & 5 & 5 & 10 & 12 & 8 & 0.2465 & 0.415 & 1 & Frequency \\
10 & 9 & 13 & 12 & 9 & 9 & 7 & 8 & 11 & 7 & 0.888137 & 0.954 & 0.979 & Block frequency \\
15 & 12 & 6 & 13 & 7 & 10 & 9 & 4 & 5 & 14 & 0.0742 & 0.1407 & 1 & Cumulative sums \\
10 & 8 & 11 & 7 & 4 & 14 & 10 & 10 & 13 & 8 & 0.4580 & 0.6528 & 0.979 & Runs \\
12 & 10 & 9 & 8 & 4 & 11 & 7 & 11 & 12 & 11 & 0.6602 & 0.8148 & 0.989 & Longest run \\
12 & 9 & 6 & 8 & 12 & 10 & 7 & 8 & 9 & 1 & 0.6833 & 0.8302 & 1 & Rank \\
5 & 11 & 13 & 13 & 6 & 14 & 8 & 7 & 11 & 7 & 0.2757 & 0.4533 & 0.989 & FFT \\
2 & 8 & 12 & 4 & 11 & 12 & 16 & 10 & 14 & 6 & 0.01724 & 0.029 & 1 & Nonoverlapping template \\
6 & 13 & 14 & 7 & 13 & 13 & 9 & 8 & 6 & 6 & 0.2328 & 0.3964 & 0.989 & Overlapping template \\
7 & 11 & 15 & 7 & 8 & 9 & 11 & 5 & 14 & 8 & 0.3074 & 0.4925 & 0.989 & Universal \\
8 & 11 & 8 & 11 & 17 & 7 & 14 & 7 & 5 & 7 & 0.1184 & 0.2204 & 1 & Approximate entropy \\
13 & 6 & 6 & 3 & 3 & 3 & 3 & 6 & 5 & 6 & 0.0519 & 0.1122 & 0.963 & Random excursions \\
5 & 2 & 8 & 4 & 7 & 7 & 3 & 7 & 1 & 10 & 0.0856 & 0.1808 & 0.981 & Random excursion variant \\
8 & 11 & 13 & 7 & 9 & 11 & 11 & 15 & 2 & 8 & 0.1528 & 0.2775 & 0.989 & Serial \\
5 & 16 & 10 & 9 & 11 & 9 & 11 & 8 & 6 & 10 & 0.3973 & 0.593 & 0.989 & Linear complexity \\
\hline
\end{tabular}

$\mathrm{C} 1$ to $\mathrm{C} 10$ is 95 . When the sequence is sufficiently large, the $P$ - value should be averagely distributed in the 10 intervals, and the frequency of occurrence in each interval is counted as $F_{i}$. If the number of sequences is $m, F_{1}+F_{2}+\cdots+F_{10}=m$, then the statistics are calculated as

$$
\chi^{2}=\sum_{i=1}^{10} \frac{\left(F_{i}-(m / 10)\right)^{2}}{m / 10}
$$

Then, calculate the $P-$ value $_{T}$ :

$$
P-\text { value }_{T}=\operatorname{igamc}\left(\frac{9}{2}, \frac{\chi^{2}}{2}\right) \text {. }
$$

If $P-$ value $_{T}>0.001$, then the $P$ - values are subject to uniform distribution; in other words, the sequence is random; otherwise, it is nonrandom.

The performance of the tests is successful for the proposed two systems. From Tables 2 and 3, all $P$ - values given in the statistical tests are larger than 0.01 , and condition (a) is met. The relevant proportions are within the acceptance interval [0.9601, 1.0298], so condition (b) is also satisfied. Furthermore, all $P-$ value $_{T} s$ are greater than 0.001 . For simplicity, the Nonoverlapping template (test no. 9 in Tables 2 and 3 ) is considered as an example. The distribution of $P-$ values for nonoverlapping template is uniform (as shown in Figures 14(a) and 14(b)). Other 14 tests display a similar uniformity. Hence, condition (c) is satisfied as well. It can be concluded that Case 1 and Case 2 systems are eligible as pseudorandom generators according to the NIST standard.

\subsection{Field Programmable Gate Array Implementation for Two} Cases. The parameters of analog circuit components are affected by temperature, aging, and other environmental factors, and chaos system is extremely sensitive to the initial value of the system, and using analog circuit to realize chaotic systems is quite limited. Hence, the digital circuits are better to realize chaotic systems. Generally, there are two implementation methods: one is based on Euler algorithm and Runge-Kutta discrete algorithm to write the underlying hardware code to realize the chaotic system and the other method is using MATLAB's DSP Builder library to build the 


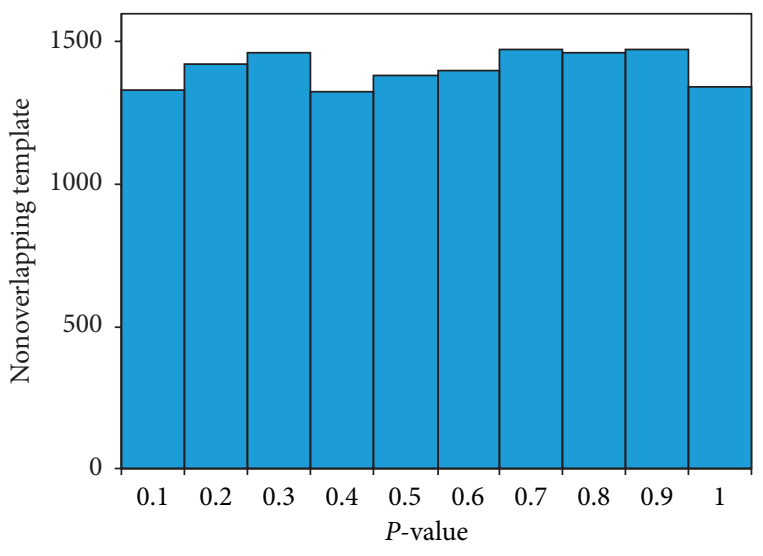

(a)

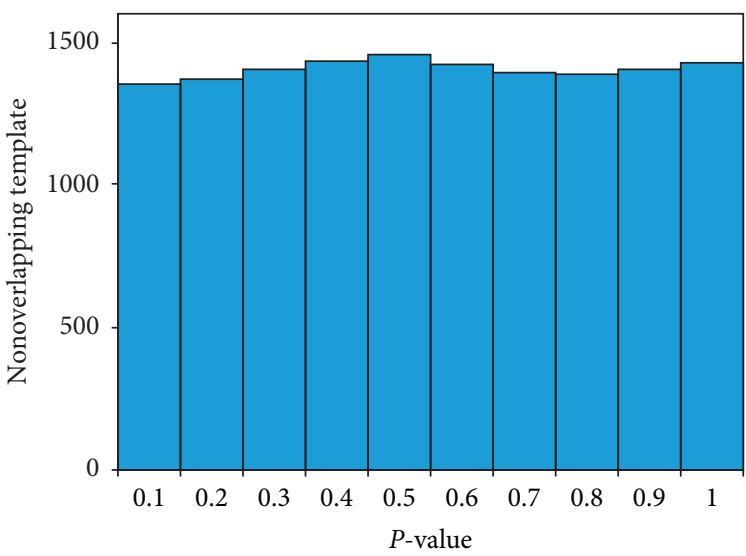

(b)

FIgURe 14: Probability distribution of $P$ - value for the nonoverlapping template. (a) Case 1 system. (b) Case 2 system.

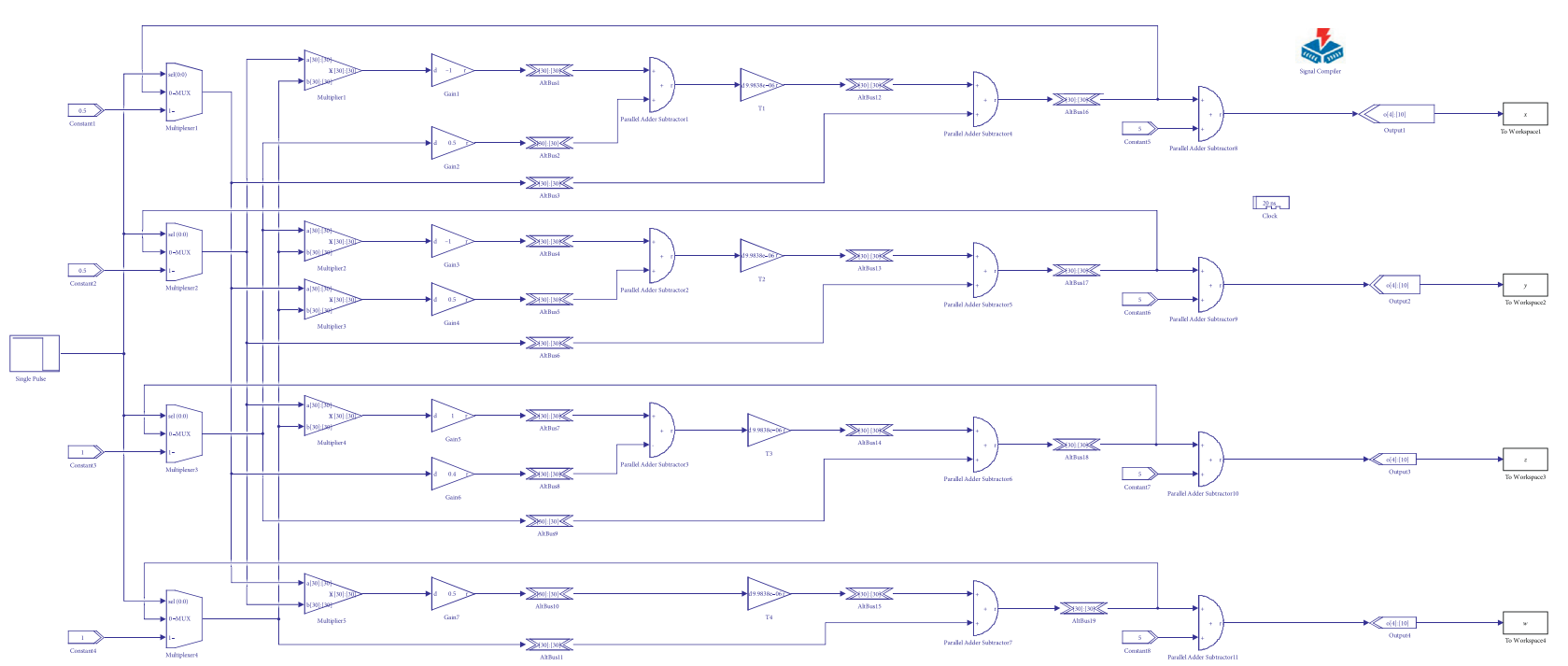

FIgURE 15: DSP Builder model of Case 1 and Case 2 systems.

chaotic digital circuit and automatically generate Verilog Language. This paper adopts the latter method. Because the conservative chaotic system is highly sensitive to initial values, parameters, and computational errors, its hardware implementation is more difficult than common dissipative chaotic systems. In this section, the implementation of the proposed Case 1 and Case 2 systems in FPGA is discussed. To build DSP builder model (Figure 15), these two systems are first discretized.

For Case 1: setting $(h, d, b, c)=(-1,2.5,2,0.2)$, one obtains

$$
\left\{\begin{array}{l}
x(n+1)=-\Delta T \cdot y(n) \cdot w(n)+0.5 \cdot \Delta T \cdot z(n)+x(n) \\
y(n+1)=-\Delta T \cdot z(n) \cdot w(n)+0.5 \cdot \Delta T \cdot x(n) \cdot w(n)+y(k), \\
z(n+1)=\Delta T \cdot y(n) \cdot w(n)-0.4 \cdot \Delta T \cdot x(n)+z(n), \\
w(n+1)=0.5 \cdot \Delta T \cdot x(n) \cdot y(n)+w(n) .
\end{array}\right.
$$

For Case 2: setting $(h, g, a, e)=(0.5,10,6,5.5)$, one obtains

$$
\left\{\begin{array}{l}
x(n+1)=0.5 \Delta T \cdot y(n) \cdot w(n)+10 \cdot \Delta T \cdot z(n)+x(n), \\
y(n+1)=6 \Delta T \cdot z(n) \cdot w(n)+5.5 \cdot \Delta T \cdot x(n) \cdot w(n)+y(k), \\
z(n+1)=-6 \Delta T \cdot y(n) \cdot w(n)-10 \cdot \Delta T \cdot x(n)+z(n), \\
w(n+1)=-6 \cdot \Delta T \cdot x(n) \cdot y(n)+w(n) .
\end{array}\right.
$$

Secondly, the chaotic digital circuits are built in Matlab/ Simulink/DSP Builder, with the fixed step of $10^{-2} \mathrm{~s}$, initial values $(0.5,0.5,1,1),(1,1,1,1)$, and sampling time $T=10^{-5} \mathrm{~s}$. The system models (Figure 15) are converted to the Verilog Hardware Description Language and finally synthesized and compiled to the register transfer level code. The process of compiling and downloading to FPGA core board is completed through QuartusII 15.1. Table 4 shows the consumption of system resources. The occupancy rate of total logical elements is $12 \%$, and the occupancy rate of total memory bits is less than $1 \%$. The field programmable gate array board is used to perform digital implementation, and a 14-bit dual-channel DA converter AD764 is used to convert 
TABLe 4: System resource consumption.

\begin{tabular}{lccc}
\hline Logic elements & Multiplier elements & Registers & Memory bits \\
\hline 17260 & 48 & 11932 & 5690 \\
\hline
\end{tabular}

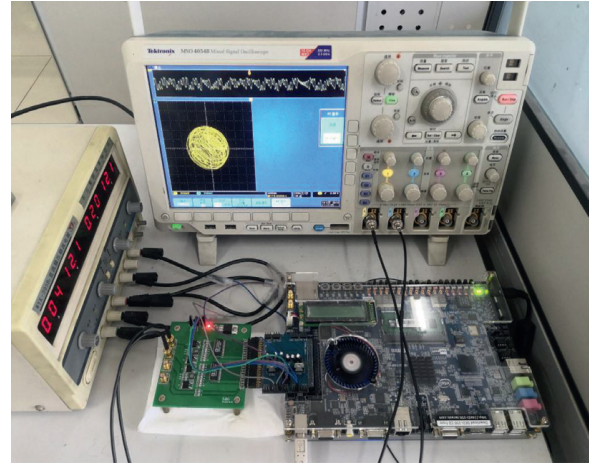

(a)

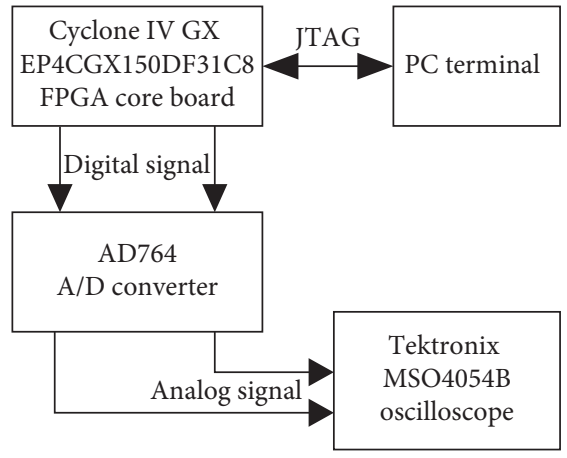

(b)

Figure 16: (a) The experimental circuit of the chaotic orbits. (b) Hardware design.

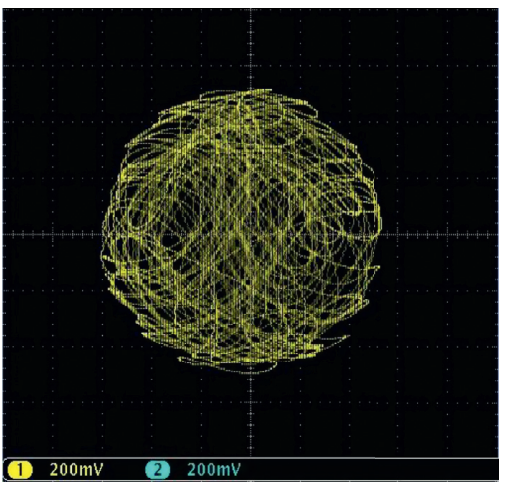

(a)

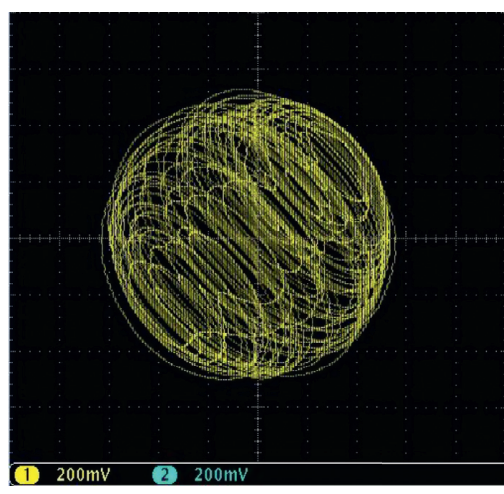

(b)

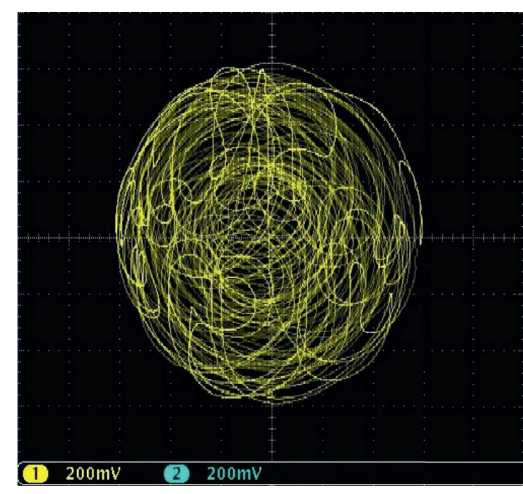

(c)

Figure 17: Phase portraits of Case 1 chaotic system using FPGA. (a) $x-y$ plane. (b) $x-z$ plane. (c) $y$-w plane.

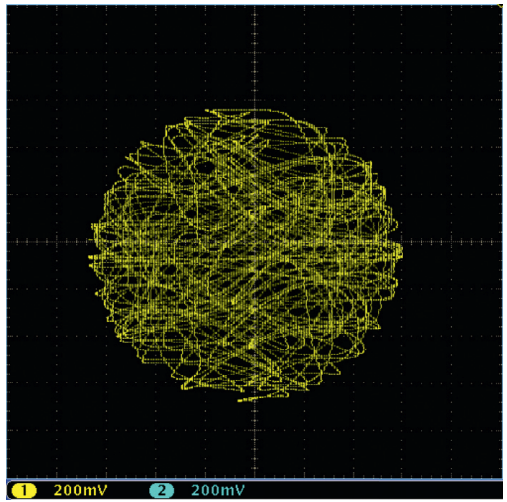

(a)

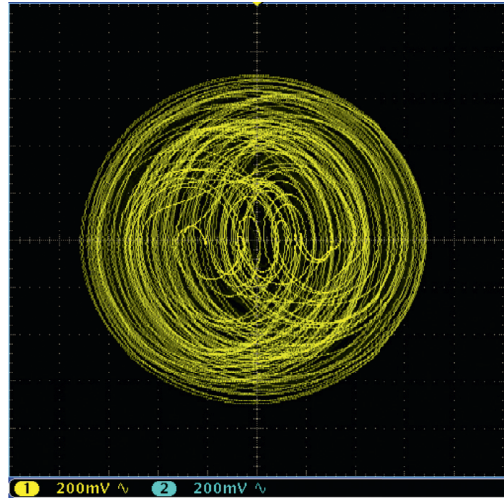

(b)

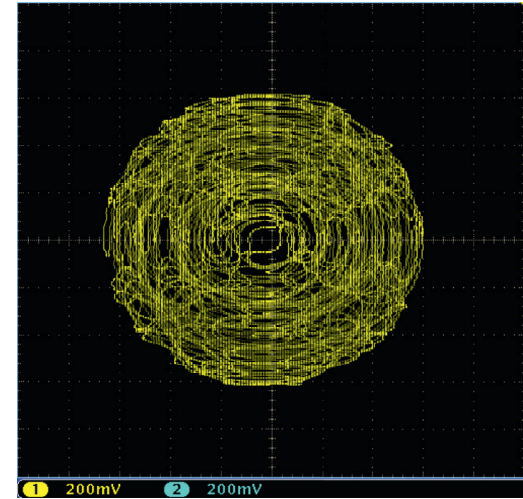

(c)

Figure 18: Phase portraits of Case 2 chaotic system using FPGA. (a) $x-y$ plane. (b) $x-z$ plane. (c) $y-w$ plane.

time series. The converted data are displayed by a digital oscilloscope (Tektronix MSO4054B) to demonstrate phase portraits (Figure 16(a)). The hardware design of the circuit implementation is shown in Figure 16(b). In order to compare the computer simulation with the digital circuit results, the phase diagrams of Case 1 and Case 2 generated by the field programmable gate array are shown in Figures 17 and 18 , respectively. It can be seen that the phase diagrams of 
these two systems are consistent with the results of computer simulation.

\section{Conclusion}

This paper constructed two HCCSs by two methods. The first method is based on 4D Euler equations, which generate chaos by breaking the Casimir energy conservation. The second method is based on a proposed method of constructing a class of Hamiltonian conservative chaotic system and then choosing the appropriate functions to construct HCCS. The dynamic behaviors of the proposed two HCCSs have been investigated based on the equilibrium point analysis, singular orbits, and energy analysis. The NIST tests indicate that the proposed two new HCCSs have good application values in information encryption. Finally, a pseudorandom chaotic signal generator is implemented based on the field programmable gate array digital development platform, which is useful for potential engineering applications.

\section{Data Availability}

The data used to support the conclusions of this research article can be computed by the relative equations and parameters given in the article using ODE45 solver in MATLAB and are available from the corresponding author upon request.

\section{Conflicts of Interest}

The authors declare that they have no conflicts of interest.

\section{Authors' Contributions}

Enzeng Dong and Guanghan Liu contributed equally to this study.

\section{Acknowledgments}

This study was partially supported by the Natural Science Foundation of China (no. 61603274), Natural Science Foundation of Tianjin (no. 18JCYBJC87700), South African National Research Foundation Grants (nos. 112108 and 112142), and South African National Research Foundation Incentive Grant (no. 114911).

\section{References}

[1] E. N. Lorenz, "Deterministic nonperiodic flow," Journal of the Atmospheric Sciences, vol. 20, no. 2, pp. 130-141, 1963.

[2] G. Chen and T. Ueta, "Yet another chaotic attractor," International Journal of Bifurcation and Chaos, vol. 9, no. 7, pp. 1465-1466, 1999.

[3] J. Lü and G. Chen, "A new chaotic attractor coined," International Journal of Bifurcation and Chaos, vol. 12, no. 3, pp. 659-661, 2002.

[4] S. Cang, Y. Li, and Z. Wang, "Single crystal-lattice-shaped chaotic and quasi-periodic flows with time-reversible symmetry," International Journal of Bifurcation and Chaos, vol. 28, no. 13, 2018.
[5] S. Cang, A. Wu, Z. Wang, and Z. Chen, "On a 3-D generalized Hamiltonian model with conservative and dissipative chaotic flows," Chaos, Solitons \& Fractals, vol. 99, pp. 45-51, 2017.

[6] E. Mahmoud, "Dynamics and synchronization of new hyperchaotic complex Lorenz system," Mathematical and Computer Modelling, vol. 55, no. 7-8, pp. 1951-1962, 2012.

[7] S. Nikolov and S. Clodong, "Occurrence of regular, chaotic and hyperchaotic behavior in a family of modified Rossler hyperchaotic systems," Chaos, Solitons \& Fractals, vol. 22, no. 2, pp. 407-431, 2004.

[8] E. Dong, Z. Chen, Z. Chen et al., "Pitchfork bifurcation and circuit implementation, of a novel Chen hyper-chaotic system," China Physics B, vol. 21, no. 3, pp. 92-100, 2012.

[9] S. Cang, G. Qi, and Z. Chen, "A four-wing hyper-chaotic attractor and transient chaos generated from a new 4-D quadratic autonomous system," Nonlinear Dynamics, vol. 59, no. 3, pp. 515-527, 2010.

[10] S. Cang, A. Wu, Z. Wang, W. Xue, and Z. Chen, "Birth of oneto-four-wing chaotic attractors in a class of simplest threedimensional continuous memristive systems," Nonlinear Dynamics, vol. 83, no. 4, pp. 1987-2001, 2016.

[11] E. Dong, Z. Liang, and S. Du, "Topological horseshoe analysis on a four-wing chaotic attractor and its FPGA implement," Nonlinear Dynamics, vol. 83, no. 1-2, pp. 623-630, 2016.

[12] E. Dong, Z. Zhang, M. Yuan, Y. Ji, X. Zhou, and Z. Wang, "Ultimate boundary estimation and topological horseshoe analysis on a parallel 4D hyperchaotic system with any number of attractors and its multi-scroll," Nonlinear Dynamics, vol. 95, no. 4, pp. 3219-3236, 2019.

[13] S. Cang, Y. Li, Z. Kang, and Z. Wang, "A generic method for constructing $\mathrm{n}$-fold covers of $3 \mathrm{D}$ conservative chaotic systems," Chaos: An Interdisciplinary Journal of Nonlinear Science, vol. 30, no. 3, Article ID 0331, 2020.

[14] S. Vaidyanathan and C. Volos, "Advances and applications in chaotic systems," Studies in Computational Intelligence, Springer, Berlin, Germany, 2016.

[15] V. Patidar, N. K. Pareek, and K. K. Sud, "A new substitutiondiffusion based image cipher using chaotic standard and logistic maps," Communications in Nonlinear Science and Numerical Simulation, vol. 14, no. 7, pp. 3056-3075, 2009.

[16] S. Cang, A. Wu, Z. Wang, and Z. Chen, "Four-dimensional autonomous dynamical systems with conservative flows: twocase study," Nonlinear Dynamics, vol. 89, no. 4, pp. 24952508, 2017.

[17] E. Dong, M. Yuan, S. Du, and Z. Chen, "A new class of Hamiltonian conservative chaotic systems with multistability and design of pseudo-random number generator," Applied Mathematical Modelling, vol. 73, no. 9, pp. 40-71, 2019.

[18] E. Ott, Chaos in Dynamical Systems, Cambridge University Press, Cambridge, UK, 2002.

[19] M. Henon and C. Heiles, "The applicability of the third integral of motion: some numerical experiments," The Astronomical Journal, vol. 6973 pages, 1964.

[20] B. Grammaticos, "Nonlinear dynamics: integrability, chaos and patterns," Journal of Physics A: Mathematical and General, vol. 37, no. 5, pp. 1949-1950, 2004.

[21] G. Qi, "Modelings and mechanism analysis underlying both the 4D Euler equations and Hamiltonian conservative chaotic systems," Nonlinear Dynamics, vol. 95, no. 3, pp. 2063-2077, 2018.

[22] W. G. Hoover, "Remark on "some simple chaotic flows"” Physical Review E, vol. 51, no. 1, pp. 759-760, 1995.

[23] S. Vaidyanathan and C. Volos, "Analysis and adaptive control of a novel 3-d conservative no-equilibrium chaotic system," Archives of Control Sciences, vol. 25, no. 3, pp. 333-353, 2015. 
[24] G. Mahmoud and M. Ahmed, "Analysis of chaotic and hyperchaotic conservative complex nonlinear systems," Miskolc Mathematical Notes, vol. 18, pp. 315-326, 2017.

[25] V. I. Arnor'd, "Kolmogorov's hydrodynamic attractors," Proceedings Mathematical Physical Sciences, vol. 434, pp. 1922, 1991.

[26] G. Qi and X. Liang, "Mechanism and energy cycling of the Qi four-wing chaotic system," International Journal of Bifurcation and Chaos, vol. 27, no. 12, p. 1750180, 2017.

[27] G. Qi and J. Zhang, "Energy cycle and bound of Qi chaotic system," Chaos, Solitons \& Fractals, vol. 99, pp. 7-15, 2017.

[28] S. Wiggins, Introduction to Applied Nonlinear Dynamical Systems and Chaos, Springer, New York, NY, USA, 2nd edition, 2003.

[29] G. Qi, J. Hu, and Z. Wang, "Modeling of a Hamiltonian conservative chaotic system and its mechanism routes from periodic to quasiperiodic, chaos and strong chaos," Applied Mathematical Modelling, vol. 78, pp. 350-365, 2019.

[30] S. Cang, Y. Li, R. Zhang, and Z. Wang, "Hidden and selfexcited coexisting attractors in a Lorenz-like system with two equilibrium points," Nonlinear Dynamics, vol. 95, no. 1, pp. 381-390, 2019.

[31] J. Zhang, W. Tang, and W. Tang, “A novel bounded 4D chaotic system," Nonlinear Dynamics, vol. 67, no. 4, pp. 2455-2465, 2012.

[32] E. Dong, M. Yuan, C. Zhang, J. Tong, Z. Chen, and S. Du, "Topological horseshoe analysis, ultimate boundary estimations of a new 4D hyperchaotic system and its FPGA implementation," International Journal of Bifurcation and Chaos, vol. 28, no. 7, Article ID 1850081, 2018.

[33] T. G. Shepherd, "Symmetries, conservation laws, and Hamiltonian structure in geophysical fluid dynamics," Advances in Geophysics, vol. 32, no. 2, pp. 287-338, 1990.

[34] P. Roe, "Characteristic-based schemes for the euler equations," Annual Review of Fluid Mechanics, vol. 18, no. 1, pp. 337-365, 2003.

[35] J. Marsden and T. Ratiu, Introduction to Mechanics and Symmetry: A Basic Exposition of Classical Mechanical Systems, Springer, Berlin, Germany, 2nd edition, 2002.

[36] D. Rosin, Dynamics of Complex Autonomous Boolean Networks, Springer, Berlin, Germany, 2015.

[37] H. Kokubu and R. Roussarie, "Existence of a singularly degenerate heteroclinic cycle in the Lorenz system and its dynamical consequences: part I," Journal of Dynamics and Differential Equations, vol. 16, no. 2, pp. 513-557, 2004.

[38] A. Wolf, J. B. Swift, H. L. Swinney, and J. A. Vastano, "Determining Lyapunov exponents from a time series," Physica D: Nonlinear Phenomena, vol. 16, no. 3, pp. 285-317, 1985.

[39] A. Wolf, "Quantifying chaos with Lyapunov exponents," Nonlinear Science: Theory and Applications, Manchester University Press, Manchester, UK, 1986.

[40] A. Rukhin, National Institute of Standards and Technology, and Technology Administration U S Department of Commerce, A Statistical Test Suite for Random and Pseudorandom Number Generators for Cryptographic Applications, NIST, Gaithersburg, MD, USA, 2001. 